\title{
FHL3 Contributes to EMT and Chemotherapy Resistance Through Inhibiting Ubiquitination of Slug and Activating TGF $\beta /$ Smad-Independent Pathways in Gastric Cancer
}

\section{Guodong Cao}

First Affiliated Hospital of Anhui Medical University

\section{Pengping Li}

Hangzhou Xiaoshan No 1 People's Hospital

\section{Qiang Sun}

Xuzhou Medical University

\section{Sihan Chen}

First Affiliated Hospital of Anhui Medical University

\section{Xin Xu}

First Affiliated Hospital of Anhui Medical University

\section{Xiaobo He}

First Affiliated Hospital of Anhui Medical University

\section{Zhenyu Wang}

Hangzhou Xiaoshan No 1 People's Hospital

\section{Peng Chen}

First Affiliated Hospital of Anhui Medical University

\section{Maoming Xiong ( $\nabla$ ayfyxmm@163.com )}

First Affiliated Hospital of Anhui Medical University

\section{Bo Chen}

First Affiliated Hospital of Anhui Medical University

\section{Research}

Keywords: EMT, Chemotherapy resistance, FHL3, Ubiquitination, Gastric cancer

Posted Date: October 9th, 2020

DOI: https://doi.org/10.21203/rs.3.rs-87249/v1

License: (c) (i) This work is licensed under a Creative Commons Attribution 4.0 International License. 
Loading [MathJax]/jax/output/CommonHTML/jax.js

Page 2/28 


\section{Abstract}

Background: Gastric cancer presents high risk of metastasis and chemotherapy resistance. Hence, the mechanistic understanding of the tumor metastasis and chemotherapy resistance is quietly important.

Methods: TCGA database and clinical samples are used for exploring the role of FHL3 in disease progression and prognosis. The roles of FHL3 in metastasis and chemotherapy resistance are explored in vitro and in vivo by siRNA or shRNA treatment. Finally, we explore the FHL3-mediated EMT and chemotherapy resistance.

Results: mRNA and protein level of FHL3 is significantly up-regulated in gastric cancer tissues when compares with it in adjacent tissue. Higher expression level of FHL3 companies with worse overall survival in gastric cancer. OPH resistance cells show higher level of FHL3 and mesenchymal phenotype. Knockdown of FHL3 slightly inhibits the cell growth, while it obviously sensitizes the chemotherapy in vivo and in vitro. In addition, down-regulation of FHL3 decreases the mesenchymal markers, such as Slug, Snail, Twist1, and vimentin, while increases the epithelial marker E-cadherin. For mechanism study, FHL3 knockdown down-regulates the expression level or activity of MAPK/ERK pathway and

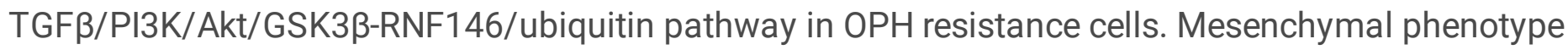
cells hold higher level of MDR1, and the FHL3 knockdown reverts the MDR1 in this type cell.

Conclusion: FHL3 is a risk of disease progression in gastric cancer. MAPK and PI3K pathways were activated when FHL3 induces EMT and drug resistance process, but the TGF $\beta /$ Smad -dependent pathway did not participate in the process. FHL3 competitively bond the ubiquitin complex (slug/GSK3 $3 /$ RNF146) with slug, inhibit ubiquitination of Slug.

\section{Background}

Gastric cancer (GC) is the fifth most common malignancy and accounts for the third leading cause of cancer death [1]. Although prediction about the prognosis of GC mainly according to the lymph node and distant metastasis status, evidence shows that conventional staging criteria fail to sufficiently differentiate the prognostic characteristics of GC [2]. Due to the high risk of lymph node metastasis and distant metastasis, about $65 \%$ cases of GC appear tumor recurrence and disease progression after surgical treatment, which leads the 5-year survival of GC down to about $35 \%$ [3]. So, many patients with or without evidence of tumor metastasis after resection surgery have to receive chemotherapy. As for the first-line drug for GC, 5-FU, and oxaliplatin (OPH) are usually appear tolerance and resistance during treatment, and that leads to unsatisfactory clinical outcomes [4]. Thus, the new prognostic biomarkers for GC are urgently needed to improve the early diagnosis and radical cure rate of gastric cancer. Meantime, the mechanistic understanding of the tumor metastasis and chemotherapy resistance should be improved to develop novel treatment targets for advanced gastric cancer.

As a wildly recognized mechanism, epithelial-mesenchymal transition (EMT) plays an irreplaceable in the all know, reduction of E-cadherin is a hallmark in EMT 
process [5-7]. Besides, both of the down-regulation of other epithelial genes, such as Z0-1 and occludin, and the up-regulation of mesenchymal genes, such as N-cadherin, vimentin, a-SMA, and fibronectin, are the characteristics in the EMT process [5-8]. EMT-associated transcriptional factors (EMT-TFs), including Slug, Snail, Twist, and Zeb, directly regulate the expression of E-cadherin by binding to the promotor sequence of E-cadherin, which named E-box [5-8]. Most signaling pathways interfere with the regulation of E-cadherin through EMT-TFs, such as smad-independent TGF- $\beta$ pathway, smad-dependent TGF- $\beta$ pathway [9-11]. For the former one, the activated TGF- $\beta$ receptor can initiate the $W n t / \beta$-catenin pathway, $\mathrm{PI} 3 \mathrm{~K} / \mathrm{Akt}$ pathway, and MAPK/ERK pathways to up-regulate the level of EMT-TFs [11]. For the later one, activated TGF- $\beta$ activates smad-complex/EMT-TFs to up-regulate the level of EMT-TFs [9].

Interestingly, the recent studies point out that EMT is not necessary for tumor invasion and metastasis, but is responsible for chemotherapy resistance $[12,13]$. And, the mechanism of EMT-associated chemotherapy is complex. Cancer stem cell (CSC) is a feature in mesenchymal phenotype tumor cells, which is closely related to chemotherapy resistance [14-16]. In addition, some regulatory factors directly related to EMT can promote chemotherapy resistance. EMT-TFs including Twist, Snail, and FOXC2 regulate chemoresistance by increasing the expression of ATP-binding cassette (ABC) transporters in breast cancer, which is important for chemoresistance $[17,18]$. And Slug can inhibit the activity of caspase-9 to endow chemoresistance to tumor cells [19]. However, single inhibition of EMT-TFs has few effects in reverting the chemoresistance. And it may be caused by other pathways which participate in the regulation of EMT process. According to previous studies, some EMT-associated pathways are also important in chemoresistance, such as PI3K/AKT pathway, MAPK pathway, and hypoxia pathway, through which chemotherapy resistance is probably induced by up-regulation of $A B C$ transporters and down-regulation of apoptosis [20-25]. Thus, the mechanism of EMT-associated chemotherapy resistance is complicated and unknown, and more works are needed to figure out the mechanism of EMT-associated chemotherapy resistance.

LIM-only protein FHLs, including FHL1, FHL2, and FHL3, are characterized by evolutionarily conserved LIM domains and one conserved LIM superfamily domain [26]. FHLs are reported as the transcriptional factors, which participate in lots of signaling pathways. Simply, FHL1-2 are reported in regulation of TGF $\beta /$ Smad-independent pathway, such as PI3K/Akt, Wnt/ $\beta$-catenin, and MAPK/ERK pathways, through which FHLs participate in EMT process and chemo- radio- therapy resistance in pancreatic cancer, breast cancer, and osteosarcoma $[27,7,26]$. Besides, FHL1-3 are considered as inhibitors of cell cycle checkpoints $\mathrm{CDC} 25$, through which they lead to radioresistance in pancreatic cancer and HeLa cell line [28]. In addition, some studies show that FHLs can interact with ER-a to make tumor progression in breast cancer $[29,30]$. Another study shows FHL2 can directly interact with epithelial phenotype marker ZO-1 to promote tumor invasion in breast cancer [31]. And in our previous study, FHL3 also acts as a regulator in ubiquitin degradation process of EMT-TFs through Akt/GSK3 $\beta /$ ubiquitin pathway in pancreatic cancer [7]. However, some previous studies have suggested that FHL1-3 perform as a tumor repressor in lung cancer, liver cancer, and breast cancer. As far as now, here without enough evidence to reveal the role of FHL3 in chemoresistance and metastasis in gastric cancer.

Loading [MathJax]/jax/output/CommonHTML/jax.js

Page 4/28 
In fact, our previous study has shown that FHL3 is an important role in the regulation of EMT. In this study, we explore the potential relationship between FHL3 and EMT and chemotherapy resistance in gastric cancer. Simply, we explore the role of FHL3 in disease progression and overall prognosis by investigating TCGA database and clinical samples in GC. Then, we explored the effects of FHL3 on proliferation, metastasis, and chemotherapy resistance in GC cells in vitro and in vivo experiments. In addition, we investigate the underlying mechanisms of FHL3-mediated EMT process and FHL3-mediated chemotherapy resistance in GC cell lines.

\section{Material And Methods}

\section{Gastric cancer sample preparation}

This study was approved by The First Affiliated Hospital of Anhui Medical University Review Board and the ethics committees of Anhui Medical University. 120 matched paraffin-embedded tumor tissue sections and 16 paired fresh frozen tissue were collected. All patients who underwent total or partial gastrectomy at the First Affiliated Hospital of Anhui Medical University from 2013 to 2016. All patients with gastric cancer were confirmed by at least two pathologists. Follow-up time was estimated from the date of surgical treatment to that of an event (i.e., patient death or tumor recurrence) or withdrawal.

\section{Bioinformatic analysis}

Gene profiles of GC and non-tumor adjacent tissues based on microarray were downloaded from Gene Expression Omnibus database (GEO; https://www.ncbi.nlm.nih.gov/geo/). TCGA and GTEx RNA sequencing FPKM data of GC, non-tumor adjacent tissues, and normal stomach tissues were downloaded from the UCSC Xena database (https://xenabrowser.net/hub/). The proteomic data of GC and non-tumor adjacent tissues were downloaded from PRIDE Archive under the accession number PXD011821. KEGG pathway analysis was performed using R clusterProfiler package. Gene Set Enrichment Analysis (GSEA) was performed by the JAVA program using gene sets collection (c2.cp.v7.1.symbols.gmt) from the MsigDB.

\section{Cell culture and $\mathrm{OPH}-$ resistance cell lines}

Gastric cancer cell lines (SGC-7901, MGC-823, AGS, and N87) and normal gastric epithelial cells (GES-1) were obtained from the cell bank of the Chinese Academy of Science. All cell lines were cultured in RPMI1640 medium (Gibco, USA). All culture media were supplemented with $10 \%$ fetal calf serum and 100 units/mL penicillin and streptomycin. Gastric cancer cell lines (HGC and SGC) are firstly detected the IC50 of $\mathrm{OPH}$. Secondly, the cells are screened in this dose-treatment of $\mathrm{OPH}$ for at least 3 generates. Then, the $\mathrm{OPH} \mathrm{IC50}$ of those cells is re-detected, and the cells are screened in the this IC50 OPH again. The screen cycle is performed at least 6 times.

\section{Western blot analysis}


Total protein extraction: Cells were harvested with a cytology brush, lysed with RIPA lysis buffer (SigmaAldrich, USA) supplemented with a phosphorylase and protease inhibitor mixture (Thermo Fisher Scientific, USA), and quantified by a BCA assay.

The standard detailed experimental process used for Western blotting was the same as that in our previous study. Western blot bands were quantified with ImageJ software (NIH, USA).

\section{MTT assay}

The cells in the logarithmic phase were plated onto 96-well plates at a density of 5000 cells per well in $200 \mu \mathrm{l}$ of culture medium and incubated for 24,48 , and $96 \mathrm{~h}$ at $37{ }^{\circ} \mathrm{C}$ with $5 \% \mathrm{CO}_{2}$. A volume of $20 \mu \mathrm{l}$ MTT solution ( $5 \mathrm{mg} / \mathrm{ml}$; Solarbio Science \& Technology, Beijing, China) was added into each well and incubated for another $4 \mathrm{~h}$. The MTT solution was then removed and $100 \mu \mathrm{l}$ dimethyl sulfoxide (Sigma) was added to each well. The relative optical density (OD) was measured at $570 \mathrm{~nm}$ and the experiment was repeated three times.

\section{Immunofluorescence}

Briefly, $2.5 \times 10^{4}$ cells were seeded in 24 -well plates for $24 \mathrm{~h}$, fixed with $4 \%$ paraformaldehyde, permeabilized with $0.5 \%$ Triton X-100, and blocked with $5 \%$ BSA (Sigma-Aldrich) for $1 \mathrm{~h}$ at $37^{\circ} \mathrm{C}$. The samples were incubated with a primary antibody (FHL3, 1:200, Proteintech) overnight at $4{ }^{\circ} \mathrm{C}$.

Subsequently, the cells were washed with PBS and incubated with secondary antibodies for $1 \mathrm{~h}$ at room temperature before being washed again. Finally, the nuclei were stained with $15 \mu \mathrm{l}$ DAPI (Sigma-Aldrich, USA) before detection with a fluorescence microscope (Carl Zeiss, Germany).

\section{Colony forming efficiency assay}

Firstly, cells were seeded in 6-well plates at a density of 1500 cells per well and incubated in $37^{\circ} \mathrm{C}$ for 10 days. Then cells were washed with PBS and fixed with $1 \mathrm{~mL} 4 \%$ formaldehyde solution. Then $1 \mathrm{~mL}$ crystal violet staining solution was added and washed with PBS for 3 times after 30 minutes.

\section{Live \& death staining}

Cells were seeded in a 6-well plate and then the cells were transfected with three FHL3 siRNA for $48 \mathrm{~h}$. After that, $\mathrm{AO}$ and $\mathrm{PI}$ solutions were added into plates and maintained for $0.5 \mathrm{~h}$ before observed by an inverted fluorescent microscope.

\section{Wound-healing assay}

Cells were cultured in a six-well plate and grown to $90 \%$ confluence in $2 \mathrm{ml}$ of culture medium. A $10 \mu \mathrm{l}$ plastic tip was used to create an artificial wound. Images were taken at 0,24 , and $48 \mathrm{~h}$ after scratching. The cell mobility $=(0 \mathrm{~h}$ width- the indicated time points width $) / 0 \mathrm{~h}$ width $\times 100 \%$.

\section{Migration and Invasion Assay}


Transwell (Corning Life Sciences, Bedford, MA, USA) and Matrigel invasion (BD Biosciences) were used to assess GC cell migratory and invasive abilities respectively. For migration assays, $1 \times 10^{5}$ cells were added to $200 \mu \mathrm{l}$ serum-free DMEM in the upper chamber and the lower chamber was filled with $600 \mu \mathrm{l}$ culture medium. After incubation at $37^{\circ} \mathrm{C}$ in an atmosphere containing $5 \% \mathrm{CO}_{2}$ for $24 \mathrm{~h}$, the non-migrated cells were carefully removed with a wet cotton swab. Finally, the cells were stained with Giemsa (Sigma, USA) for 10 min followed by imaging and counting under an inverted microscope (100x magnification). The cell invasion assay was carried out similarly, while the invasion assay was coated with Matrigel before cells were seeded on the membrane.

\section{Subcutaneous tumor model}

Male nude mice (4 to 6 weeks old) obtained from the SLAC (Shanghai, China) were randomly divided into two groups. A total of $1 \times 10^{6}$ cells (FHL3-NC and FHL3-SH1 cells) in $100 \mu$ l PBS were injected into the subcutaneous. 4 weeks later, the mother tumors are harvested to make the same volume transplanted tumors. Then the FHL3-NC-derived and FHL3-SH1-derived transplanted tumors are transplanted into mice. The tumor volume is investigated every day. All mice were sacrificed, and the tumors are harvested to determine the tumor volume $\left(\mathrm{MaA} \times \mathrm{MiA}^{2} / 2 ; \mathrm{MaA}=\right.$ Major axis, MiA=Minor axis), followed by processing into sections for HE staining and Ki67 staining.

\section{Orthotopic transplantation model}

Simply, the transplanted tumors are transplanted into the subserosa of stomach in SCID mice. 4 weeks later, mice are executed to investigate the volume and weight of tumors.

\section{Lung metastasis model}

Simply, $1 \times 10^{6}$ cells are injected into the tail vein of SCID mice after 4 weeks, and the lungs are harvested for frozen sections to investigate the metastatic tumors by FITC-tag.

\section{Immunoprecipitation and recombination plasmid system}

Primers of Slug, FHL3, and RNF146 are inserted into plasmid pcDNA 3.1(-) (Addgene). Briefly, cDNA templates are synthesized through PrimeScript RT Reagent Kit (TaKaRa, China); CDS of genes are amplified with PrimeSTAR ${ }^{\circledR}$ GXL DNA Polymerase (TaKaRa, China); Products are purified by SanPrep Column DNA Gel Extraction Kit (Sangon Biotech, China); The purified products and plasmids are treated with restriction endonuclease (Xho1, EcoR5, and Xba1 comes from NEB, USA) respectively; Recombination of plasmids are performed through homologous recombination with Hieff CloneTM Plus One Step Cloning Kit (Yeasen Biotech, China). Cells were transplanted into 6 wells plates for $24 \mathrm{~h}$ followed by transfection of RP for different times with Hieff TransTM Liposomal Transfection Reagent (Yeasen Biotech, China) to find out the best transfection efficiency, according to the manufacturer's instructions.

A total of $1 \times 10^{7}$ cells were harvested with a cytology brush and lysed with RIPA lysis buffer (Yeasen upernatant, followed by adding magnetic beads (Anti-Myc, 
Anti-HA Bimake and Anti-Flag) with continuous slight mixing at $4{ }^{\circ} \mathrm{C}$ for $24 \mathrm{~h}$. Then, the magnetic beads were isolated with a magnet (Bimake), followed by washing with TBS. Finally, the products were boiled before being dissolved in 5x SDS (Yeasen) for 5-10 minutes for Western blot assays.

\section{Statistical analysis}

All experimental data are presented as the mean \pm SD. Statistical Package for the Social Sciences version 21.0 (SPSS Inc., USA) was used for the statistical analyses. ANOVA, paired t-test, Chi-square $\left(x^{2}\right)$ test, and a nonparametric test (Mann-Whitney $U$ ) were used for statistical analysis in different situations.

Statistical significance was defined as $p<0.05\left({ }^{*} P<0.05 ; * * P<0.01 ; * \star * P<0.001\right)$. All histograms and curves were constructed with GraphPad Prism 6 software (GraphPad Software, La Jolla, CA, USA).

\section{Results}

\section{FHL3 is a disease progression factor and prognosis prediction biomarker in GC}

To investigate the clinical relevance of FHL3 in GC, we systematically analyze multiple publicly available gene expression datasets (The Cancer Genome Atlas/Genotype Tissue Expression (TCGA/GTEx), GSE13861, GSE13911, GSE19826, GSE29998, GSE54129, and GSE63089), which contain > 1,000 gastric cancer patients. We notice that FHL3 mRNA expression is remarkably up-regulated in GC (Fig. 1a, b, d, e, f and $\mathrm{g}, P<0.001)$. In line with this observation, FHL3 protein expression is also significantly elevated in GC by proteogenomic analysis of Beijing dataset which contains 58 paired gastric tumor samples and nontumor adjacent tissues (PXD011821, Fig. 1h, $P<0.001$ ). Furthermore, we explore the role of FHL3 in the progression of GC. We perform Kaplan-Meier analyses in both TCGA and KM-plotter cohorts, and the survival curves show that patients with higher expression level of FHL3 have shorter overall survival time than those with lower expression levels in TCGA $(\mathrm{HR}=1.40,95 \% \mathrm{Cl}=1.15-1.51, P=0.039, \mathrm{Fig} .1 \mathrm{i})$ and KM-plotter cohorts $(\mathrm{HR}=1.55,95 \% \mathrm{Cl}=1.31-1.84, P<0.001$, Fig. 1j).

To confirm this observation, we examine the FHL3 level in clinical gastric tumor samples. According to the IHC staining result, up-regulated FHL3 expression is positively associated with the tumor TNM stage (Fig. 2a). Consistently, the expression of FHL3 is dramatically up-regulated GC tissues when compares with it in non-tumor adjacent tissues in both 120 matched paraffin-embedded tumor tissue sections and 16 paired fresh frozen tissue (Fig. $2 \mathrm{~b}, \mathrm{~d}$ and e, $P<0.05$ ). In addition, according to the FHL3 expression and the follow-up time of $120 \mathrm{GC}$ patients, Kaplan-Meier analysis indicates that higher expression of FHL3 leads to worse prognosis in GC patients $(P<0.001$, Fig. 2c). In 16 paired GC tissue, higher expression level of FHL3 always accompanies with worse TNM stage (Fig. 2f, $P=0.0484$ ).

The correlations between FHL3 and clinicopathological parameters were shown in Table 1. And, there is no significant difference in FHL3 expression in different groups such as gender $(P=0.67)$, age $(P=0.72)$, tumor size $(P=0.50)$, tumor location $(P=0.66)$, depth of invasion $(P=0.94)$ and lymph node metastasis 
$(P=0.77)$. However, as Table 1 shows, higher expression level of FHL3 accompanies with differentiation $(P=0.009)$, metastasis $(P=0.002)$ and TNM stage $(P=0.039)$.

Table 1

Association between clinicopathological parameters and FHL3 in 120 cases of gastric cancer

\begin{tabular}{|c|c|c|c|c|c|}
\hline & & $\begin{array}{l}\text { Total } \\
\text { patients }\end{array}$ & $\begin{array}{l}\text { FHL3- } \\
\text { positive }\end{array}$ & $\begin{array}{l}\text { FHL3- } \\
\text { negative }\end{array}$ & $\begin{array}{l}P \\
\text { value }\end{array}$ \\
\hline \multirow[t]{2}{*}{ Sex } & Male & 80 & 21 & 59 & 0.67 \\
\hline & Female & 40 & 12 & 28 & \\
\hline \multirow[t]{2}{*}{ Age } & $<60 y$ & 50 & 13 & 37 & 0.72 \\
\hline & $>60 y$ & 70 & 20 & 50 & \\
\hline \multirow[t]{2}{*}{ Tumor size } & $<3 \mathrm{~cm}$ & 23 & 5 & 18 & 0.50 \\
\hline & $>3 \mathrm{~cm}$ & 97 & 28 & 69 & \\
\hline \multirow[t]{2}{*}{ Differentiation } & Well/moderate & 101 & 23 & 78 & 0.009 \\
\hline & Poor & 19 & 10 & 9 & \\
\hline \multirow[t]{2}{*}{ Tumor location } & $\begin{array}{l}\text { Upper/ } \\
\text { Medium }\end{array}$ & 62 & 17 & 60 & 0.66 \\
\hline & Low & 58 & 12 & 31 & \\
\hline \multirow[t]{2}{*}{ Depth of invasion } & $\mathrm{T} 1+\mathrm{T} 2$ & 26 & 7 & 19 & 0.94 \\
\hline & $\mathrm{T} 3+\mathrm{T} 4$ & 94 & 26 & 68 & \\
\hline \multirow{2}{*}{$\begin{array}{l}\text { Lymph node } \\
\text { metastasis }\end{array}$} & NO & 24 & 5 & 19 & 0.77 \\
\hline & $\mathrm{N} 1+\mathrm{N} 2+\mathrm{N} 3$ & 96 & 28 & 68 & \\
\hline \multirow[t]{2}{*}{ Metastasis } & MO & 112 & 27 & 85 & 0.002 \\
\hline & M1 & 8 & 6 & 2 & \\
\hline \multirow[t]{2}{*}{ TNM stage } & $I+I I$ & 35 & 5 & 30 & 0.039 \\
\hline & III + IV & 85 & 28 & 57 & \\
\hline
\end{tabular}

At the same time, the prognostic value of FHL3 and clinical parameters are evaluated using univariate and multivariate analysis, respectively. In univariate analysis, lymph node metastasis (Hazard ratio $=1.43$, $95 \% \mathrm{Cl}: 1.15-1.78, P=0.001$, Table 2 ) and FHL3 (Hazard ratio $=2.29,95 \% \mathrm{Cl}: 1.45-3.63, P<0.001$, Table 2) are the risk factors of disease progression in GC. The multivariate Cox proportional hazards model shows lymph node metastasis (Hazard ratio $=1.47,95 \% \mathrm{Cl}: 1.09-1.98, P=0.011$, Table 2 ) and expression level of FHL3 (Hazard ratio $=2.06,95 \%$ Cl: $1.23-3.42, P=0.005$, Table 2 ) are the independent 
Table 2

Univariate analysis and multivariate analysis of overall survival in 120 gastric cancer patients

\begin{tabular}{|c|c|c|c|c|c|c|}
\hline Variables & $\begin{array}{l}\text { Univariate } \\
\text { analysis }\end{array}$ & & & $\begin{array}{l}\text { Multivariate } \\
\text { analysis }\end{array}$ & & \\
\hline & $\begin{array}{l}\text { Hazard } \\
\text { Ratio }\end{array}$ & $95 \% \mathrm{Cl}$ & $P$ value & Hazard Ratio & $95 \% \mathrm{Cl}$ & $P$ value \\
\hline Sex & 1.20 & $\begin{array}{l}0.77- \\
1.89\end{array}$ & 0.40 & 1.12 & $\begin{array}{l}0.68- \\
1.85\end{array}$ & 0.65 \\
\hline Age & 0.98 & $\begin{array}{l}0.97- \\
1.00\end{array}$ & 0.08 & 0.98 & $\begin{array}{l}0.96 \\
-1.00\end{array}$ & 0.033 \\
\hline Tumor size & 1.03 & $\begin{array}{l}0.57- \\
1.86\end{array}$ & 0.93 & 0.70 & $\begin{array}{l}0.34- \\
1.42\end{array}$ & 0.32 \\
\hline Differentiation & 0.85 & $\begin{array}{l}0.58- \\
1.25\end{array}$ & 0.41 & 1.20 & $\begin{array}{l}0.72 \\
-2.00\end{array}$ & 0.49 \\
\hline Tumor location & 0.99 & $\begin{array}{l}0.89- \\
1.11\end{array}$ & 0.90 & 0.93 & $\begin{array}{l}0.83- \\
1.05\end{array}$ & 0.25 \\
\hline Depth of invasion & 1.06 & $\begin{array}{l}0.83- \\
1.34\end{array}$ & 0.66 & 1.13 & $\begin{array}{l}0.73- \\
1.76\end{array}$ & 0.58 \\
\hline $\begin{array}{l}\text { Lymph node } \\
\text { metastasis }\end{array}$ & 1.43 & $\begin{array}{l}1.15- \\
1.78\end{array}$ & 0.001 & 1.47 & $\begin{array}{l}1.09- \\
1.98\end{array}$ & 0.011 \\
\hline Metastasis & 1.55 & $\begin{array}{l}0.72- \\
3.37\end{array}$ & 0.27 & 1.37 & $\begin{array}{l}0.28- \\
6.61\end{array}$ & 0.70 \\
\hline TNM stage & 1.32 & $\begin{array}{l}0.95- \\
1.83\end{array}$ & 0.10 & 0.97 & $\begin{array}{l}0.47- \\
1.99\end{array}$ & 0.94 \\
\hline FHL3 expression & 2.29 & $\begin{array}{l}1.45- \\
3.63\end{array}$ & 0.0004 & 2.06 & $\begin{array}{l}1.24- \\
3.42\end{array}$ & 0.005 \\
\hline
\end{tabular}

\section{FHL3 knockdown reduces OPH resistance in vitro}

In order to investigate the relationship between the expression of FHL3 and chemotherapy in GC, we first screen out the FHL3-high-expression GC cell lines. We detect the FHL3 expression level in a normal gastric cell line (GES-1) and several gastric cancer cell lines (SGC, HGC, AGS and N87). As the results show, FHL3 is high expression in all GC cell lines in contrast with GES-1, while SGC and HGC hold the first and second highest level of FHL3 respectively (SGC-7901 > HGC > AGS > N87 > GES-1, Fig. 3a). Then we make OPH-resistance (OPH-R) cell lines in SGC and HGC cell lines. As the Fig. $2 \mathrm{~b}$ and $\mathrm{c}$ show, OPH-R cell lines hold higher tolerance in OPH (HGC: $P<0.01$, SGC: $P<0.05$ ). Whereafter, we silence the FHL3 by lentivirus and verify the knockdown efficiency by western blot (WB) and immunofluorescence (IF) in those $\mathrm{OPH}-\mathrm{R}$ cell lines. Both WB and IF assays show the same result that $\mathrm{SH} 1$ is most efficient in FHL3 knockdown, and the FHL3-SH1 cell lines join our following experiments (Fig. 3d-f). Colony formation assay and live \& death staining assay are performed to detect the effect of FHL3-knockdown on OPH 
growth ability with the treatment of OPH and NS (Fig. $3 g$, i and j), while the FHL3 knockdown slightly decreases the colony unit formation $(P<0.05)$. Encouragingly, the combination treatment (FHL3 knockdown and OPH) restricts the tumor growth of about $80 \%$ in OPH-R HGC/SGC cell lines (Fig. $3 \mathrm{~g}$, i and j, $P<0.01$ ). Collectively, FHL3 knockdown significantly enhances the $\mathrm{OPH}$-induced tumor growth inhibition rate about 1 -fold as comparing with the single OPH treatment group (Fig. g, i and j, $P<0.05$ ). In the live \& death staining assay, our study shows that obviously less live cells (green) and more death cells (red) in the combination treatment group, when compares with other groups (Fig. $3 \mathrm{~h}, \mathrm{k}$ and I, $P<0.01$ ).

\section{Down-regulation of FHL3 reverts the EMT phenotype and restricts tumor metastasis}

To investigate the association of FHL3 and EMT, we analyze the data in TCGA. And our study find that the expression level of FHL3 is negatively correlated to the expression level of E-cadherin $(r=-0.403, P<$ 0.001 , Fig. $\left.4 \mathrm{a}_{1}\right)$, and passively correlated to $\mathrm{N}$-cadherin $\left(\mathrm{r}=0.116, P=0.025\right.$, Fig. $\left.4 \mathrm{a}_{2}\right)$, vimentin $(\mathrm{r}=0.421$, $P<0.001$, Fig. $\left.4 a_{3}\right)$ and EMT-TFs Twistl $\left(r=0.347\right.$, $P<0.001$, Fig. $\left.4 a_{4}\right)$, Snail $\left(r=0.353, P<0.001\right.$, Fig. $\left.4 a_{5}\right)$ and Slug $\left(r=0.358, P<0.001\right.$, Fig. $\left.4 a_{6}\right)$. These data imply that FHL3 may contribute to EMT process. In Fig. $3 b_{1-7}$, our study shows that FHL3 knockdown significantly up-regulates the expression of E-cadherin and ZO-1 more than 1-fold, while it down-regulated the Vimentin, Slug, and Snail more than $50 \%$ (Fig. $4 \mathrm{~b}_{1}$ $\left.{ }_{-7}, P<0.01\right)$. However, the Twist1 gets slight changes with the treatment of FHL3 knockdown. In the following experiments, our study finds that FHL3 knockdown restricts the cell migration more than $50 \%$ in HGC cell lines, and about $50 \%$ in SGC cell lines (Fig. $4 c$ and $e_{1}, P<0.05$ ). In the wound healing assay, we get similar results (Fig. $4 d_{2}$ and $e_{2}$ ).

\section{FHL3 knockdown reduces OPH resistance and metastasis in subcutaneous/orthotopic stomach tumor bearing-model and lung metastasis model}

During our study in vivo, we find subcutaneous tumors growth speed of OPH-R HGC cells is slower in single FHL3-SH1 group and single OPH group as compared with FHL3-NC group (Fig. 5a and b, $P<0.05$ ). Interestingly, combination treatment (FHL3-SH1 and $\mathrm{OPH}$ ) significantly inhibits the tumor growth when compares with FHL3-SH1 + NS and FHL3-NC + OHP groups $(P<0.01$, Fig. 5a-b). At the end of the test, we detect the tumor volume and weight in vitro. As the Fig. 5c-d show, combination treatment increases the tumor growth inhibition about 1-fold when compares with single OPH treatment or single FHL3knockdown treatment in OPH-R HGC subcutaneous tumor model $(P<0.001)$. As shown in Fig. $5 \mathrm{e}, \mathrm{IHC}$ staining is performed, and the results show that tumors with combination treatment have a weak intensity of Ki-67, while it is obviously observed in other groups. Those results show FHL3-knockdown enhances the tumor growth inhibition of $\mathrm{OPH}$. 
Then, we validate the role of FHL3 in metastasis of gastric cancer cells in vivo. As Fig. 6a shows, 4 weeks after the orthotopic transplantation and tail intravenous injection of gastric cancer cells, the orthotopic stomach tumors and lungs were harvested for tumor detection. In our result, we find orthotopic transplantation tumor FHL3-SH1 was smaller more than 50\% as compared with FHL3-SH1 when treated with OHP ( $P<0.001$, Fig. 6b, e, f). According to the white nodules on the surface of the lungs (Fig. $6 \mathrm{c})$, we could easily find the metastasis situ in mice of the FHL3-NC + OHP group. We made slices from these lung samples and performed immunofluorescence (Fig. 6d, g), among which the green area is metastatic tumor cells from the blood cycle which come from tail vein injection or orthotopic stomach situ.

According to our observation, lung metastasis occurs in 1/5 SCID mouse in the FHL3-SH1 + OHP group (20\%), while it occurs in 4/5 SCID mice in FHL3-NC + OHP (80\%). Those results indicate us FHL3 knockdown decreases the lung metastasis from circular tumor cells more than $60 \%$.

\section{Down-regulated TGF- $\beta /$ Smad-independent pathways are features of FHL3-knockdown-induced mesenchymal- epithelial transition (MET)}

As above mentioned, EMT-associated signaling pathways and EMT markers themselves are involved in chemotherapy resistance. Thus, we explore the mechanisms of FHL3-mediated EMT and EMT-associated $\mathrm{OPH}$ resistance in GC. The results of bioinformatic analysis reveal that PI3K-Akt pathway, Cytoskeleton, focal adhesion, and ECM (extracellular matrix) play important roles in GC tumorigenesis and progression, which are dramatically correlated with FHL3 expression (Fig. 7a). Moreover, as the GSEA results show that TGF $\beta$ and MAPK signaling pathways tend to be significantly activated in patients with higher expression level of FHL3(Fig. 7b, $P<0.05$ ). So, FHL3 may promote EMT-mediated tumor invasion and EMT-associated chemotherapy resistance in GC carcinogenesis and progression through various

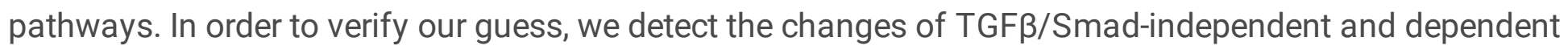
pathways during the FHL3-knockdwon-induced MET process. As the Fig. $6 \mathrm{c}_{1-4}$ show, FHL3 knockdown slightly decreases the level of ERK1/2, but greatly down-regulates the level of phosphorylated ERK $1 / 2$ (Thr202/204-ERK ${ }_{1 / 2}$, named $\mathrm{p}-\mathrm{ERK}_{1 / 2}$ here) more than $50 \%(P<0.001)$. And the ratio of $\mathrm{p}-\mathrm{ERK}_{1 / 2} / \mathrm{ERK}_{1 / 2}$ is also down-regulated by FHL3 knockdown (Fig. $7 c_{1-2}, P<0.001$ ). In addition, FHL3 knockdown also decreases the level both of Thr180/182-P38 (p-P38) and phosphorylated JNK (p-JNK) more than 50\% (Fig. $7 \mathrm{c}_{1,3}$ and ${ }_{4}, P<0.001$ ). And the ratio both of p-P38/P38 and p-JNK/JNK are also significantly downregulated (Fig. $7 \mathrm{c}_{1,3}$ and ${ }_{4}, P<0.01$ ). Those results imply that down-regulation of FHL3 leads the downregulated activity of MAPK/ERK|JNK|P38 pathways during FHL3-knockdown induced MET process. Moreover, FHL3 knockdown also decreases the level of PI3K-p85 and Tyr607-PI3K-p85 (p-PI3K) more than $50 \%$, while the ratio of $\mathrm{p}-\mathrm{PI} 3 \mathrm{~K} / \mathrm{PI} 3 \mathrm{~K}$ has not changed much (Fig. $7 \mathrm{~d}_{1-2}, P>0.05$ ). As the downstream of $\mathrm{PI} 3 \mathrm{~K}$, Ser473-Akt (p-Akt) is also significantly down-regulated more than $50 \%$ by FHL3 knockdown, while the level of Akt is up-regulated about 1.7-fold (Fig. $7 \mathrm{~d}_{1}$ and $\left.{ }_{3}, P<0.01\right)$. However, the ratio of p-Akt/Akt is still greatly down-regulated by FHL3 knockdown (Fig. $7 \mathrm{~d}_{1}$ and $\left.{ }_{3}, P<0.001\right)$. Those results imply FHL3knockdown induced down-regulated activity of PI3K and up-regulated activity of Akt. Then we find those Loading [MathJax]/jax/output/CommonHTML/jax.js on of Ser9-GSK3 $\beta$ and Ser9-GSK3 $\beta /$ GSK3 $\beta$, and the up- 
regulation of Ser217/279-GSK3 $\beta$ and Ser217/279-GSK3 $\beta /$ GSK3 $\beta$ (Fig. $7 \mathrm{~d}_{1}$ and ${ }_{4}, P<0.01$ ). According to those results, FHL3 knockdown inhibits the activity of PI3K/Akt pathway, which leads to the up-regulated activity of GSK3 $\beta$. Collectively, the activities of TGF $\beta /$ Smad-independent pathways are significantly regulated during the FHL3-knockdown induced EMT.

To the best of our knowledge, for TGF $\beta$ /smad-dependent way, smad complex directly regulates the expression level of EMT-TFs to promote EMT process. However, in our experiments, FHL3 knockdown without effect in regulation of Smad4 and p-Smad4, while it exactly decreases the level of TGF $\beta$, Thr8Smad2/3 (p-Smad2/3), Ser423/425-Smad2/3 (p-Smad2/3) and p-Smad2/3/Smad2/3. In other words, FHL3 knockdown without effect in the regulation of TGF $\beta /$ Smad-dependent pathway (Fig. $7 e_{1-4}$ ).

\section{RNF146-mediated ubiquitin participates in FHL3-induced EMT process}

In our previous study, we have found that FHL3 participates in Akt/GSK3 $\beta$ pathway-mediated ubiquitination degradation of EMT-TFs, and the E3 ligase RNF146 is firstly reported to interact with FHL3 in pancreatic cancer. Thus, we explore the role of RNF146 in FHL3-mediated stabilization of EMT-TFs in GC. As Fig. 7a shows, overexpression of RNF146 restricts the pcDNA-Slug recombination-plasmidmediated up-regulation of Slug about $50 \%$ (Fig. 8a and c, $P<0.01$ ). In addition, overexpression of FHL3 significantly enhances pcDNA-Slug recombination-plasmid-mediated up-regulation of Slug (Fig. 8a and c, $P<0.01)$. Furthermore, FHL3 overexpression eliminates the RNF146 overexpression-mediated downregulation of Slug (Fig. 8a and c, $P<0.01$ ). Then the CO-IP assay shows that RNF146 can interact both with FHL3 and Slug (Fig. 8a). Those results imply that RNF146 participates in the ubiquitination degradation of Slug, through which it interferes with EMT process.

\section{FHL3 induces chemoresistance via MDR1}

According to the previous studies, TGF $\beta /$ Smad-independent pathways containing MAPK/ERK and $\mathrm{PI3K} /$ Akt both participate in chemotherapy resistance, and those pathways have been pointed out to be probably associated with $\mathrm{OPH}$ resistance in GC basing on our above results. However, more details are needed to be finished to further figure out the FHL3-mediated chemotherapy resistance. So, we detect the level of HIF-a, caspase-3, and MDR1 in FHL3-knockdown cell lines. The results show that high-dose treatment of OPH leads up-regulation of HIF-a about 0.2-fold, caspase-3 about 0.7-fold, and MDR1 about 0.4 -fold in OPH-R FHL3-NC HGC cells, respectively (Fig. 8b, $P<0.05$ ). And the FHL3 knockdown restricts the high-dose treatment of OPH-induced up-regulation of HIF-a and MDR1 at least $50 \%$, while it upregulates the level of caspase- 3 about 0.25 -fold (Fig. $7 \mathrm{~b}, P<0.05$ ). Then, we explore the association of FHL3 and MDR1 via detecting the expression through IF on FHL3 cell line, on the sample slice through IHC. The IF shows that FHL3-NC cells have higher level of MDR1, and MDR1, and knockdown of FHL3 could decrease the expression of MDR1 (Fig. 8e). The IHC shows that advanced GC with chemotherapy resistance have higher level of FHL3 and MDR when compare with early GC (Fig. 8f). 
Despite the morbidity and mortality of GC have declined over the past decade, we still face many problems and challenges in the screening and treatment of GC. TNM staging, encompassing the depth of invasion $(T)$, lymph node metastasis $(N)$, and distant metastasis $(M)$ stages, is regarded as the most significant prognostic factor of GC. Because of tumor heterogeneity, even patients with the same pathologic stage of GC may have considerable differences in survival after complete surgical resection, indicating that prognosis cannot be accurately determined based on the current staging system alone. Even after complete resection or targeted therapy, many advanced GC patients still die of local recurrence and/or distant metastasis. Among which, tumor metastasis and chemotherapy failure are the mainly severe problem all clinical doctors have to face. Although various genes and pathways have been researched in GC, the more powerful mechanisms are still needed to resolve the metastasis and chemotherapy resistance.

Recently, LIM domain-only protein family plays pivotal roles in tumor progression, including radiotherapy resistance and metastasis $[7,28]$. Some studies showed that FHLs play as a tumor repressor in breast cancer, liver cancer, and lung cancer [32-34], while other previous studies pointed out that FHLs promotes paclitaxel resistance and radiotherapy resistance in liver cancer cells and HeLa cells respectively [28, 35], promote tumor cell growth in liver cancer, glioma and breast cancer [36, 37], and promote metastasis in breast cancer cells and pancreatic cancer [7,30]. However, the role of FHLs in gastric cancer is still unclear. In our study, we find the expression level of FHL3 is obviously up-regulated in GC both in mRNA and protein by analysis in TCGA, GTEx, and Beijing dataset $(P<0.05$, Fig. $1 \mathrm{a}-\mathrm{h})$. Meantime, the same results are gained from 16 fresh-frozen tumor tissues and 120 paraffin-embedded sections $(P<0.01)$. Then, 120 samples show higher level of FHL3 refers to lower differentiation $(P=0.009$, Table 1$)$, metastasis trend ( $P=0.002$, Table 1$)$ and worse stage of TNM $(P=0.039$, Table 1$)$ in GC. Besides, FHL3 is negatively associated with the prognosis in GC through Kaplan-Meier analysis in TCGA, KM-plotter cohorts and $120 \mathrm{GC}$ samples $(P<0.05$, Fig. $1 \mathrm{i}$, j and $2 \mathrm{c})$. Furthermore, the univariate analysis shows higher level of FHL3 accompanies with higher risk of GC progression (Table 2, HR = 2.06, $p=0.005$ ). In other words, those results suggest that FHL3 is a potential predictor of disease progression and prognosis in GC.

In the following experiments, the tumor growth is decreased 50\% in HGC and 20\% in SGC by the FHL3 knockdown (Fig. 3g, i and j). In subcutaneous tumor model, our study finds that FHL3 knockdown reduces the tumor growth $\sim 25 \%$ in tumor volume and $\sim 25 \%$ in tumor weight (Fig. $5 \mathrm{c}$ and d). Besides, FHL3 knockdown enhances the cytotoxicity of OPH both in HGC and SGC (Fig. $3 \mathrm{~g}$, i and j), which is similar to the results in subcutaneous tumor model (Fig. 5a-d). In addition, our study finds the level of FHL3 is positive with the level of Slug, Snail, Twist1, N-cadherin and vimentin, while it is negative with the level of E-cadherin (Fig. $4 \mathrm{a}_{1-6}$ ). And FHL3 knockdown obviously decreases the expression level of vimentin, Snail and Slug, and increases the level of E-cadherin and ZO-1 both in HGC and SGC (Fig. $4 \mathrm{~b}_{1-}$ 7). Meantime, migration and invasion ability are reduced by FHL3 knockdown. (Fig. 4c-e2).

However, the mechanisms of how FHL3 interferes in the regulation of tumor chemotherapy resistance Loading [MathJax]/jax/output/CommonHTML/jax.js bwledge, EMT is mainly responsible for tumor metastasis, 
and the level of E-cadherin is considered as the feature in EMT process [6, 9]. E-box, a pivotal DNA reading frame in $\mathrm{CDH} 1$ sequence, through which some transcriptional factors can directly bind to $\mathrm{CDH} 1$ to regulate the expression level of E-cadherin, such as snail1/2, twist1/2, Zeb1 and FOXC2 [9]. Besides, other cellular junction protein also paly roles in tumor metastasis, such as $\mathrm{N}$-cadherin and ZO-1. The regulation of EMT hold some recognized pathways, among which the TGF $\beta$-mediated smad-dependent and smadindependent ways are major ones $[9,6]$. For TGF $\beta /$ smad-dependent way, smad complex directly regulates the expression level of EMT-TFs to promote EMT process [9, 6]. Previous studies have showed that FHLs promote phosphorylation of $\operatorname{smad}_{2 / 3}$ and directly interact with them to lead nuclear accumulation of smad complex in liver cancer, which is important in EMT induction [32]. For TGF $\beta /$ smad-independent way, PI3K/Akt, MAPK, NF-KB, Hedgehog and Wnt/ß-catenin pathways are upstream regulators of EMTTFs [38-41]. Some studies point out that FHLs can increase the activation and transcription of Akt to promote tumor growth and progression in glioma, breast cancer, and ovarian cancer [42, 43, 30]. Other published papers show that FHLs interfere the MAPK/ERK pathway to result in radiotherapy resistance in pancreatic cancer [44]. It is reported that FHLs knockdown contributes to tumorigenesis prevention in osteosarcoma by down-regulation of Wnt/ $\beta$-catenin pathways [27]. And FHLs are also associated with hepatocarcinogenesis by activating NF-KB pathway [45]. Therefore, FHL3 is likely to regulate gastric cancer metastasis through the TGF $\beta$ /smad-independent pathway. Encouragingly, our study finds MAPK, $\mathrm{PI} 3 \mathrm{~K} / \mathrm{Akt}$ and TGF $\beta$ pathways is close to FHL3 by KEEG and GO analysis (Fig. 5a and $b$ ). And in the following experiments, FHL3 knockdown obviously down-regulates the phosphorylation level of MAPK downstream targets ERK1/2, P38 and JNK in HCG cells (Fig. 5c1-4). Besides, FHL3 knockdown obviously down-regulates the phosphorylation level of PI3K, Akt and GSK3 $\beta$ in HCG cells (Fig. $5 d_{1-4}$ ). However, FHL3 knockdown induced down-regulation of TGF $\beta$ has few effects in the phosphorylation level of Smad4. Collectively, FHL3-induced EMT is associated with the activation of MAPK/ERK/JNK/P38 and $\mathrm{PI3K} / \mathrm{Akt} / \mathrm{GSK} 3 \beta$ pathways. Furthermore, based on our previous study that FHL3 regulates the Akt/GSK3 $\beta /$ ubiquitin-Snail1/Twist1 pathway to stabilize the EMT-TFs to promote EMT process in pancreatic cancer, we explore the role of FHL3 in the regulation of ubiquitin-mediated EMT. Interestingly, we find E3 ligase RNF146 can form a complex with FHL3 and Slug, and the up-regulation of FHL3 establishes the up-regulated RNF146-induced ubiquitination degradation of Slug (Fig. 8a and b).

Well, the mechanism of chemotherapy resistance is complicated. On the one hand, $A B C$ transporters, especially for MDR1, is a central role in chemo-drug efflux to make chemotherapy resistance in gastric cancer $[46,47]$. MDR1 is directly regulated by NF-KB pathway. And hypoxia also participates in the regulation of MDR1, through which up-regulation of HIF-a can lead chemotherapy in gastric cancer [23]. On the other hand, previous studies show that the inhibition of apoptosis is also important in chemotherapy, and those apoptosis-inhibition-mediated chemotherapy is regulated by MAPK pathway and PI3K/Akt pathway [20-22]. Besides, EMT is also considered to be responsible for chemotherapy resistance in pancreatic cancer and breast cancer $[12,13]$. Nevertheless, the characteristic changes of some signaling pathways in mesenchymal phenotype may be related to the chemotherapy resistance shown by those EMT phenotype cells. Up-regulation of EMT-TFs, such as Snail and Twist1, 
regulation of EMT process $[19,17,18]$. In fact, our study finds that down-regulation of FHL3 promotes the mesenchymal-epithelial transition (MET), during which it may reduce chemotherapy resistance in HCG cells. Furthermore, our study finds MDR1 is down-regulated in FHL3-knockdown process (Fig. 8b, e). In other words, those TGF $\beta$ /smad-independent pathways are the working regulators in FHL3-mediated chemotherapy resistance.

\section{Conclusion}

Collectively, as the scheme of our hypothesis shows (Fig. 8g), FHL3 could competitively bond the ubiquitin complex (GSK3 $\beta /$ RNF146) with slug. Slug could induce the EMT process and promote cancer cell metastasis. In addition, FHL3 could induce drug resistance by activating the MAPK and PI3K pathway which may lead to the MDR1 overexpression.

\section{Abbreviations}

GC gastric cancer; OPH:oxaliplatin; EMT:epithelial-mesenchymal transition; EMT-TFs:EMT-associated transcriptional factors; CSC:cancer stem cell; ABC:ATP-binding cassette; GEO:Gene Expression Omnibus; GES-1:gastric epithelial cells; GSEA:gene set enrichment analysis; MET:mesenchymal-epithelial transition; HR:hazard ratios.

\section{Declarations}

\section{Acknowledgements}

Not applicable.

\section{Authors' contributions}

$\mathrm{CB}, \mathrm{CGD}$ and XMM designed the study; WZY and CSH contributed to date acquisition; XX and HXB contributed to the specimens' collection; CGD, LPP, SQ and XX carried out the experiments. CGD, LPP, SQ and $\mathrm{CP}$ analyzed data; CGD and CP wrote the manuscript. All authors read and approved the final version of the manuscript.

\section{Funding}

This work was supported by grants from Key Research and Development Plan Projects of Anhui Province (Project Nos.201904a07020045).

\section{Availability of data and materials}

The datasets used and analyzed during the current study are available. 


\section{Ethics approval and consent to participate}

This study is approved by The First Affiliated Hospital of Anhui Medical University Review Board and the ethics committees of Anhui Medical University. And all patients in this study have signed the informed consents.

\section{Consent for publication}

Not applicable.

\section{Competing interests}

The authors declare that they have no competing interests.

\section{References}

1. Bray F, Ferlay J, Soerjomataram I, Siegel RL, Torre LA, Jemal A. Global cancer statistics 2018 : GLOBOCAN estimates of incidence and mortality worldwide for 36 cancers in 185 countries. CA Cancer J Clin. 2018;68(6):394-424.

2. Zhang X, Song Y, Song N, Zhang L, Wang Y, Li D et al. Rankl expression predicts poor prognosis in gastric cancer patients: results from a retrospective and single-center analysis. Braz J Med Biol Res. 2018;51(3):e6265.

3. Ma DH, Li BS, Liu JJ, Xiao YF, Yong X, Wang SM et al. miR-93-5p/IFNAR1 axis promotes gastric cancer metastasis through activating the STAT3 signaling pathway. Cancer Lett. 2017;408:23-32.

4. Jingyue S, Xiao W, Juanmin Z, Wei L, Daoming L, Hong X. TFAP2E methylation promotes 5 fluorouracil resistance via exosomal miR106a5p and miR421 in gastric cancer MGC803 cells. Mol Med Rep. 2019;20(1):323-31.

5. Acloque H, Adams MS, Fishwick K, Bronner-Fraser M, Nieto MA. Epithelial-mesenchymal transitions: the importance of changing cell state in development and disease. J Clin Invest. 2009;119(6):143849.

6. Lamouille S, Xu J, Derynck R. Molecular mechanisms of epithelial-mesenchymal transition. Nat Rev Mol Cell Biol. 2014;15(3):178-96.

7. Li P, Cao G, Zhang Y, Shi J, Cai K, Zhen L et al. FHL3 promotes pancreatic cancer invasion and metastasis through preventing the ubiquitination degradation of EMT associated transcription factors. Aging (Albany NY). 2020;12(1):53-69.

8. Zeisberg M, Neilson EG. Biomarkers for epithelial-mesenchymal transitions. J Clin Invest. 2009;119(6):1429-37.

9. Gonzalez DM, Medici D. Signaling mechanisms of the epithelial-mesenchymal transition. Sci Signal. 2014;7(344):re8. 
10. Kaimori A, Potter J, Kaimori JY, Wang C, Mezey E, Koteish A. Transforming growth factor-beta1 induces an epithelial-to-mesenchymal transition state in mouse hepatocytes in vitro. J Biol Chem. 2007;282(30):22089-101.

11. Zhang YE. Non-Smad pathways in TGF-beta signaling. Cell Res. 2009;19(1):128-39.

12. Fischer KR, Durrans A, Lee S, Sheng J, Li F, Wong ST et al. Epithelial-to-mesenchymal transition is not required for lung metastasis but contributes to chemoresistance. Nature. 2015;527(7579):472-6.

13. Zheng X, Carstens JL, Kim J, Scheible M, Kaye J, Sugimoto H et al. Epithelial-to-mesenchymal transition is dispensable for metastasis but induces chemoresistance in pancreatic cancer. Nature. 2015;527(7579):525-30.

14. Mani SA, Guo W, Liao MJ, Eaton EN, Ayyanan A, Zhou AY et al. The epithelial-mesenchymal transition generates cells with properties of stem cells. Cell. 2008;133(4):704-15.

15. Singh A, Settleman J. EMT, cancer stem cells and drug resistance: an emerging axis of evil in the war on cancer. Oncogene. 2010;29(34):4741-51.

16. Singh SK, Hawkins C, Clarke ID, Squire JA, Bayani J, Hide T et al. Identification of human brain tumour initiating cells. Nature. 2004;432(7015):396-401.

17. Gottesman MM, Fojo T, Bates SE. Multidrug resistance in cancer: role of ATP-dependent transporters. Nat Rev Cancer. 2002;2(1):48-58.

18. Saxena M, Stephens MA, Pathak H, Rangarajan A. Transcription factors that mediate epithelialmesenchymal transition lead to multidrug resistance by upregulating $A B C$ transporters. Cell Death Dis. 2011;2(7):e179.

19. Chang TH, Tsai MF, Su KY, Wu SG, Huang CP, Yu SL et al. Slug confers resistance to the epidermal growth factor receptor tyrosine kinase inhibitor. Am J Respir Crit Care Med. 2011;183(8):1071-9.

20. Han Z, Hong L, Han Y, Wu K, Han S, Shen H et al. Phospho Akt mediates multidrug resistance of gastric cancer cells through regulation of P-gp, Bcl-2 and Bax. J Exp Clin Cancer Res. 2007;26(2):2618.

21. Jiao M, Nan KJ. Activation of PI3 kinase/Akt/HIF-1 a pathway contributes to hypoxia-induced epithelial-mesenchymal transition and chemoresistance in hepatocellular carcinoma. Int J Oncol. 2012;40(2):461-8.

22. Kim CW, Lu JN, Go SI, Jung JH, Yi SM, Jeong JH et al. p53 restoration can overcome cisplatin resistance through inhibition of Akt as well as induction of Bax. Int J Oncol. 2013;43(5):1495-502.

23. Liu L, Ning X, Sun L, Zhang H, Shi Y, Guo C et al. Hypoxia-inducible factor-1 alpha contributes to hypoxia-induced chemoresistance in gastric cancer. Cancer Sci. 2008;99(1):121-8.

24. Tan W, Yu HG, Luo HS. Inhibition of the p38 MAPK pathway sensitizes human gastric cells to doxorubicin treatment in vitro and in vivo. Mol Med Rep. 2014;10(6):3275-81.

25. Zhang Y, Qu X, Hu X, Yang X, Hou K, Teng Y et al. Reversal of P-glycoprotein-mediated multi-drug resistance by the E3 ubiquitin ligase $\mathrm{Cbl-b}$ in human gastric adenocarcinoma cells. J Pathol. 2009;218(2):248-55.

Loading [MathJax]/jax/output/CommonHTML/jax.js 
26. Sala S, Ampe C. An emerging link between LIM domain proteins and nuclear receptors. Cell Mol Life Sci. 2018;75(11):1959-71.

27. Brun J, Dieudonné FX, Marty C, Müller J, Schüle R, Patiño-García A et al. FHL2 silencing reduces Wnt signaling and osteosarcoma tumorigenesis in vitro and in vivo. PLoS One. 2013;8(1):e55034.

28. Xu X, Fan Z, Liang C, Li L, Wang L, Liang Y et al. A signature motif in LIM proteins mediates binding to checkpoint proteins and increases tumour radiosensitivity. Nat Commun. 2017;8:14059.

29. Xiong Z, Ding L, Sun J, Cao J, Lin J, Lu Z et al. Synergistic repression of estrogen receptor transcriptional activity by FHL2 and Smad4 in breast cancer cells. IUBMB Life. 2010;62(9):669-76.

30. Zhang F, Feng F, Yang P, Li Z, You J, Xie W et al. Four-and-a-half-LIM protein 1 down-regulates estrogen receptor a activity through repression of AKT phosphorylation in human breast cancer cell. Int J Biochem Cell Biol. 2012;44(2):320-6.

31. Zhao JL, Liang SQ, Fu W, Zhu BK, Li SZ, Han H et al. The LIM domain protein FHL1C interacts with tight junction protein ZO-1 contributing to the epithelial-mesenchymal transition (EMT) of a breast adenocarcinoma cell line. Gene. 2014;542(2):182-9.

32. Asada K, Ando T, Niwa T, Nanjo S, Watanabe N, Okochi-Takada E et al. FHL1 on chromosome X is a single-hit gastrointestinal tumor-suppressor gene and contributes to the formation of an epigenetic field defect. Oncogene. 2013;32(17):2140-9.

33. Niu C, Liang C, Guo J, Cheng L, Zhang H, Qin X et al. Downregulation and growth inhibitory role of FHL1 in lung cancer. Int J Cancer. 2012;130(11):2549-56.

34. Niu C, Yan Z, Cheng L, Zhu J, Zhang H, Xu X et al. Downregulation and antiproliferative role of FHL3 in breast cancer. IUBMB Life. 2011;63(9):764-71.

35. Zhou L, Ding L, Liu J, Zhang Y, Luo X, Zhao L et al. Four-and-a-half LIM protein 1 promotes paclitaxel resistance in hepatic carcinoma cells through the regulation of caspase-3 activation. $J$ Cancer Res Ther. 2018;14(Supplement):S767-s73.

36. Nouët Y, Dahan J, Labalette C, Levillayer F, Julien B, Jouvion G et al. The four and a half LIM-only protein 2 regulates liver homeostasis and contributes to carcinogenesis. J Hepatol. 2012;57(5):102936.

37. Sun L, Yu S, Xu H, Zheng Y, Lin J, Wu M et al. FHL2 interacts with EGFR to promote glioblastoma growth. Oncogene. 2018;37(10):1386-98.

38. Grotegut S, von Schweinitz D, Christofori G, Lehembre F. Hepatocyte growth factor induces cell scattering through MAPK/Egr-1-mediated upregulation of Snail. Embo j. 2006;25(15):3534-45.

39. Kim HJ, Litzenburger BC, Cui X, Delgado DA, Grabiner BC, Lin X et al. Constitutively active type I insulin-like growth factor receptor causes transformation and xenograft growth of immortalized mammary epithelial cells and is accompanied by an epithelial-to-mesenchymal transition mediated by NF-kappaB and snail. Mol Cell Biol. 2007;27(8):3165-75.

40. Lamouille S, Connolly E, Smyth JW, Akhurst RJ, Derynck R. TGF- $\beta$-induced activation of mTOR complex 2 drives epithelial-mesenchymal transition and cell invasion. J Cell Sci. 2012;125(Pt 
41. Yoo YA, Kang MH, Lee HJ, Kim BH, Park JK, Kim HK et al. Sonic hedgehog pathway promotes metastasis and lymphangiogenesis via activation of Akt, EMT, and MMP-9 pathway in gastric cancer. Cancer Res. 2011;71(22):7061-70.

42. Hua G, He C, Lv X, Fan L, Wang C, Remmenga SW et al. The four and a half LIM domains 2 (FHL2) regulates ovarian granulosa cell tumor progression via controlling AKT1 transcription. Cell Death Dis. 2016;7(7):e2297.

43. Li SZ, Hu YY, Zhao JL, Zang J, Fei Z, Han H et al. Downregulation of FHL1 protein in glioma inhibits tumor growth through PI3K/AKT signaling. Oncol Lett. 2020;19(6):3781-8.

44. Zienert E, Eke I, Aust D, Cordes N. LIM-only protein FHL2 critically determines survival and radioresistance of pancreatic cancer cells. Cancer Lett. 2015;364(1):17-24.

45. Dahan J, Nouët Y, Jouvion G, Levillayer F, Adib-Conquy M, Cassard-Doulcier AM et al. LIM-only protein FHL2 activates NF-KB signaling in the control of liver regeneration and hepatocarcinogenesis. Mol Cell Biol. 2013;33(16):3299-308.

46. Wallner J, Depisch D, Gsur A, Götzl M, Haider K, Pirker R. MDR1 gene expression and its clinical relevance in primary gastric carcinomas. Cancer. 1993;71(3):667-71.

47. Xu HW, Xu L, Hao JH, Qin CY, Liu H. Expression of P-glycoprotein and multidrug resistanceassociated protein is associated with multidrug resistance in gastric cancer. $\mathrm{J}$ Int Med Res. 2010;38(1):34-42.

\section{Figures}
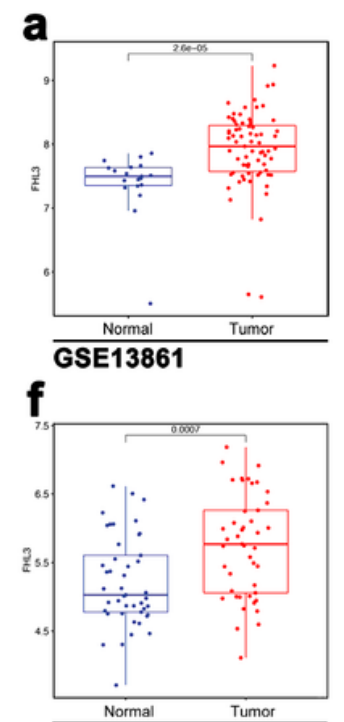

GSE63089
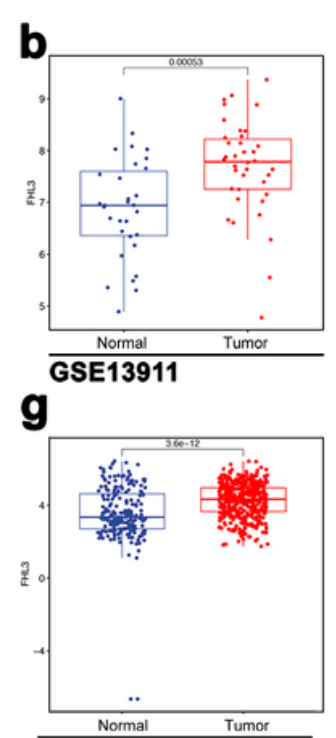

$\frac{\text { Normal }}{\text { TCGA \& GTEx }}$
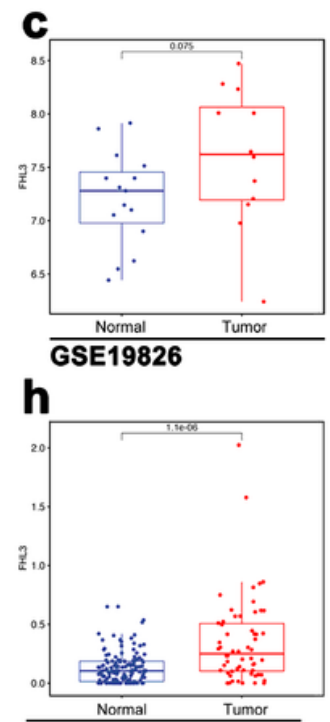

$\frac{\text { Normal }}{\text { PXD011821 }}$
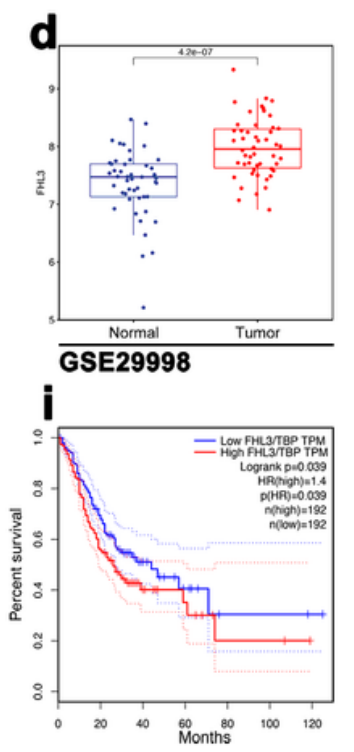
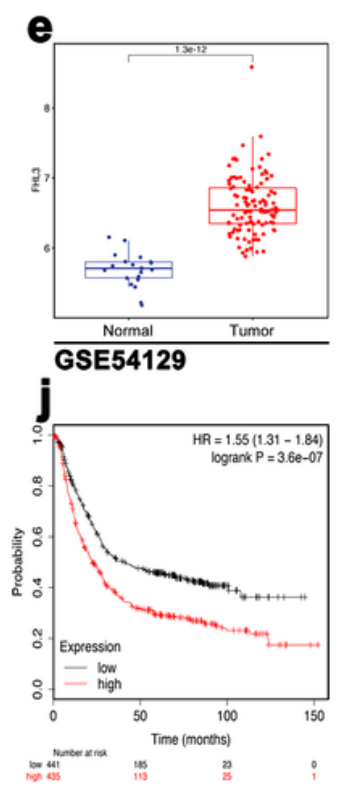

Figure 1 
FHL3 is an independent risk factor of disease progression in gastric cancer. a-h The level of FHL3 in gastric cancer is analyzed in TCGA database, GTEx, and Beijing dataset; i, j The relationship between FHL3 expression level and prognosis is performed by Kaplan-Meier analysis in TCGA, KM-plotter cohorts.

a

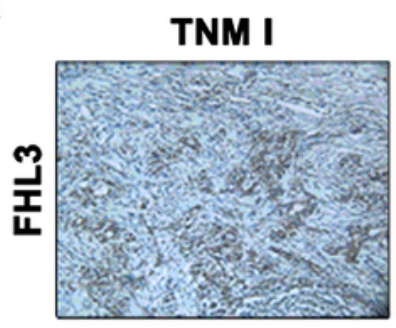

TNM II

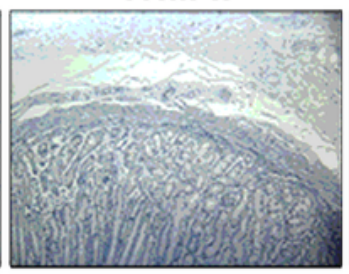

b

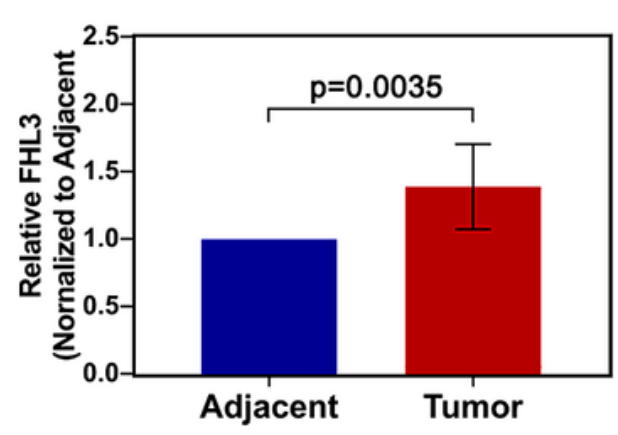

d

$$
\frac{\mathrm{I} N}{\mathrm{~T} N \mathrm{~N}} \frac{\mathrm{III}}{\mathrm{T} N} \frac{\mathrm{IV}}{\mathrm{T} N}
$$

FHL3
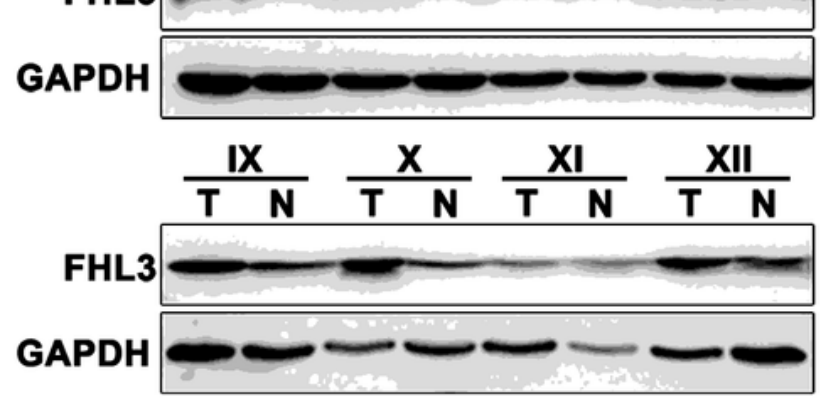

$\mathbf{e}$

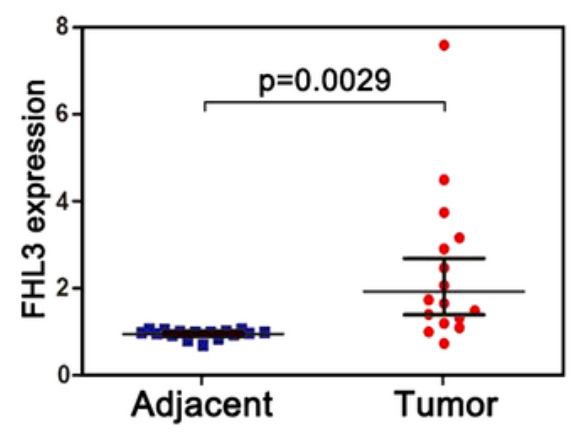

TNM III
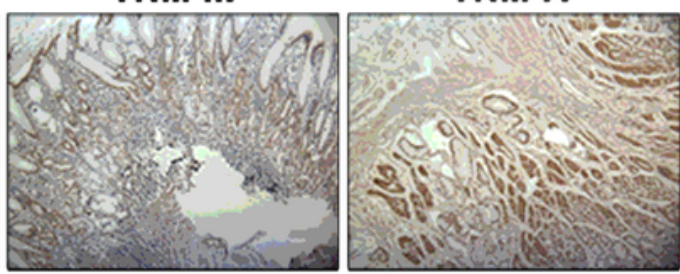

C

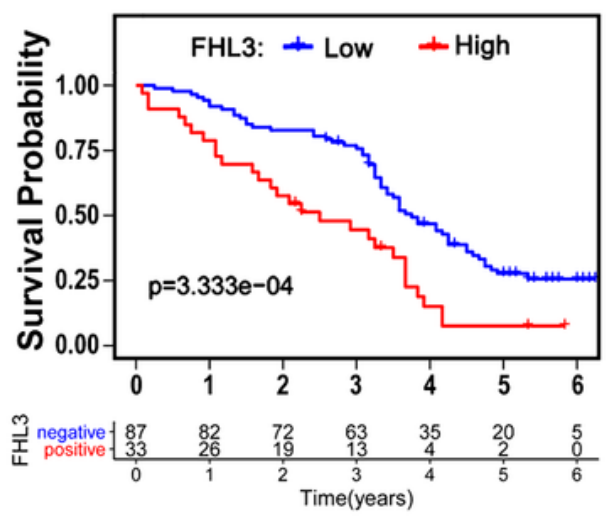

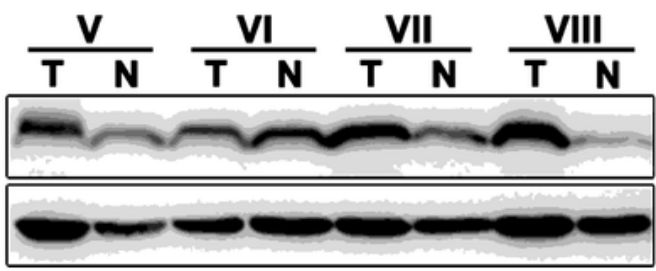

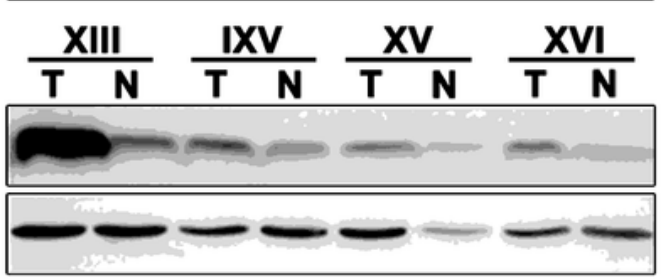

f

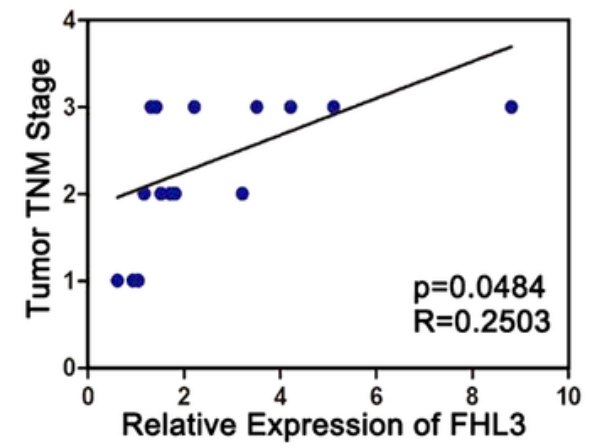

Figure 2

FHL3 is highly expressed in tumor samples and associated with survival. The level of FHL3 in gastric Loading [MathJax]/jax/output/CommonHTML/jax.js tissues and 120 paraffin-embedded sections. 

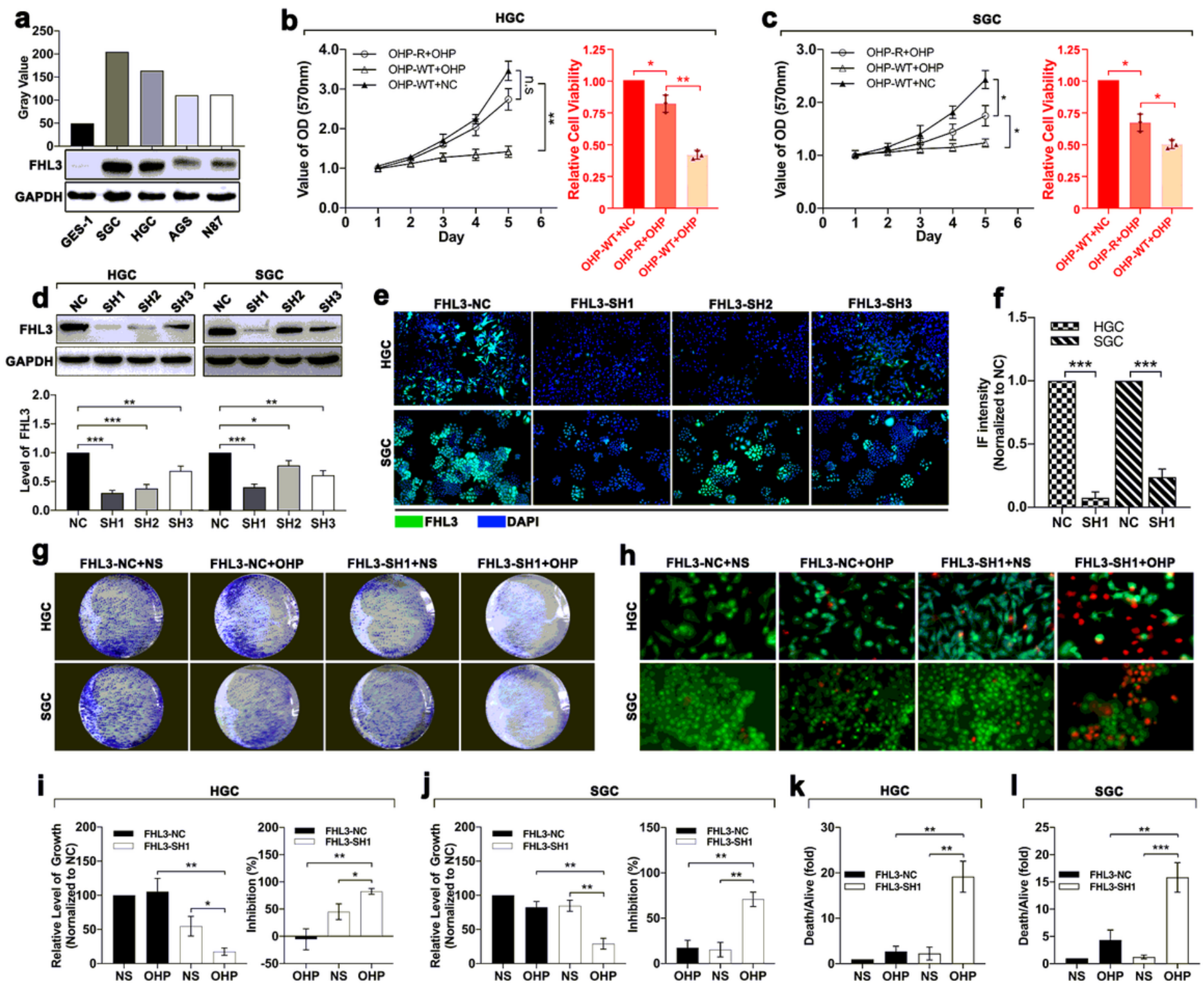

\section{Figure 3}

FHL3-knockdown significantly enhances growth inhibition of $\mathrm{OPH}$. a The gastric cancer cells are screened for FHL3-high-expression cell lines by western blot; b, $c$ The growth cures of OHP-resistance cell lines with the treatment of NS or OPH for 5 days; d-f The efficiency of the four FHL3 knockdown sequences in OPH-resistance cell lines by western blot and immunofluorescence, and the FHL3-SH1 is the most efficient sequence for down-regulation of FHL3; $\mathrm{g}$, i and $\mathrm{j}$ OPH-resistance FHL3-NC/SH1 cell lines are treated with NS or $\mathrm{OPH}$ for $48 \mathrm{~h}$, which is followed by colony formation for 14 days; $\mathrm{h}, \mathrm{k}$ and I $\mathrm{OPH}$ resistance FHL3-NC/SH1 cell lines are treated with NS or OPH for 48h, which is followed by live\&death staining assay. 

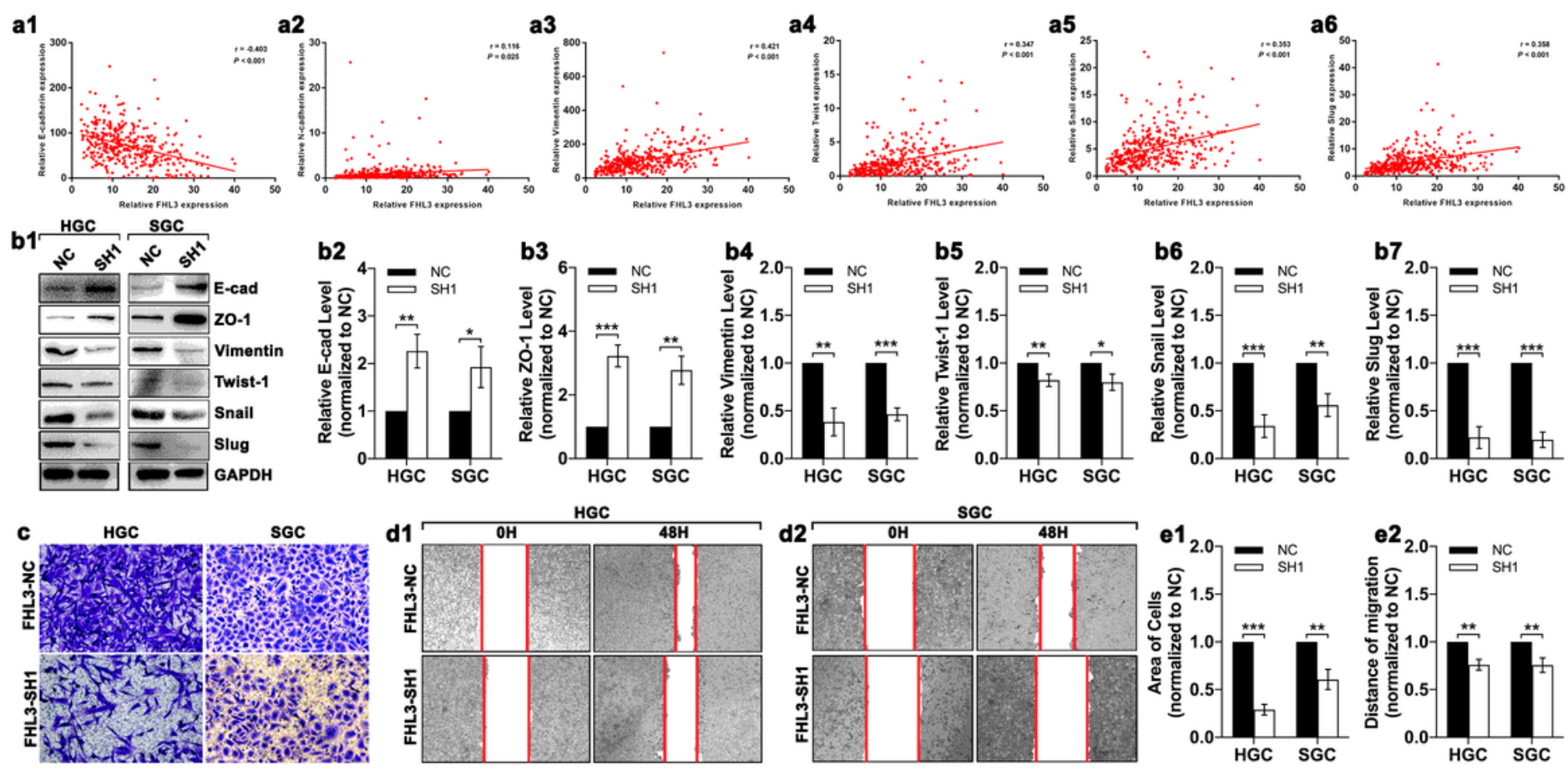

Figure 4

FHL3 is associated with the EMT process. a1-6 The relationship between expression level of FHL3 and EMT-associated transcriptional factors (Snail1, Slug, Twist,) and EMT markers ( $\mathrm{N}$-cadherin, E-cadherin and vimentin) is analyzed in TCGA database; b1-7The relationship between expression level of FHL3 and EMT-associated transcriptional factors (Snail1, Slug, Twist,) and EMT markers (N-cadherin, E-cadherin and vimentin) is verified by western blot assay in OPH-resistance FHL3-NC/SH1 cell lines; c, d1-2 and e1-2 The effects of FHL3 knockdown in migration and invasion are explored by transwell and wound healing assay in in OPH-resistance FHL3-NC/SH1 cell lines. 

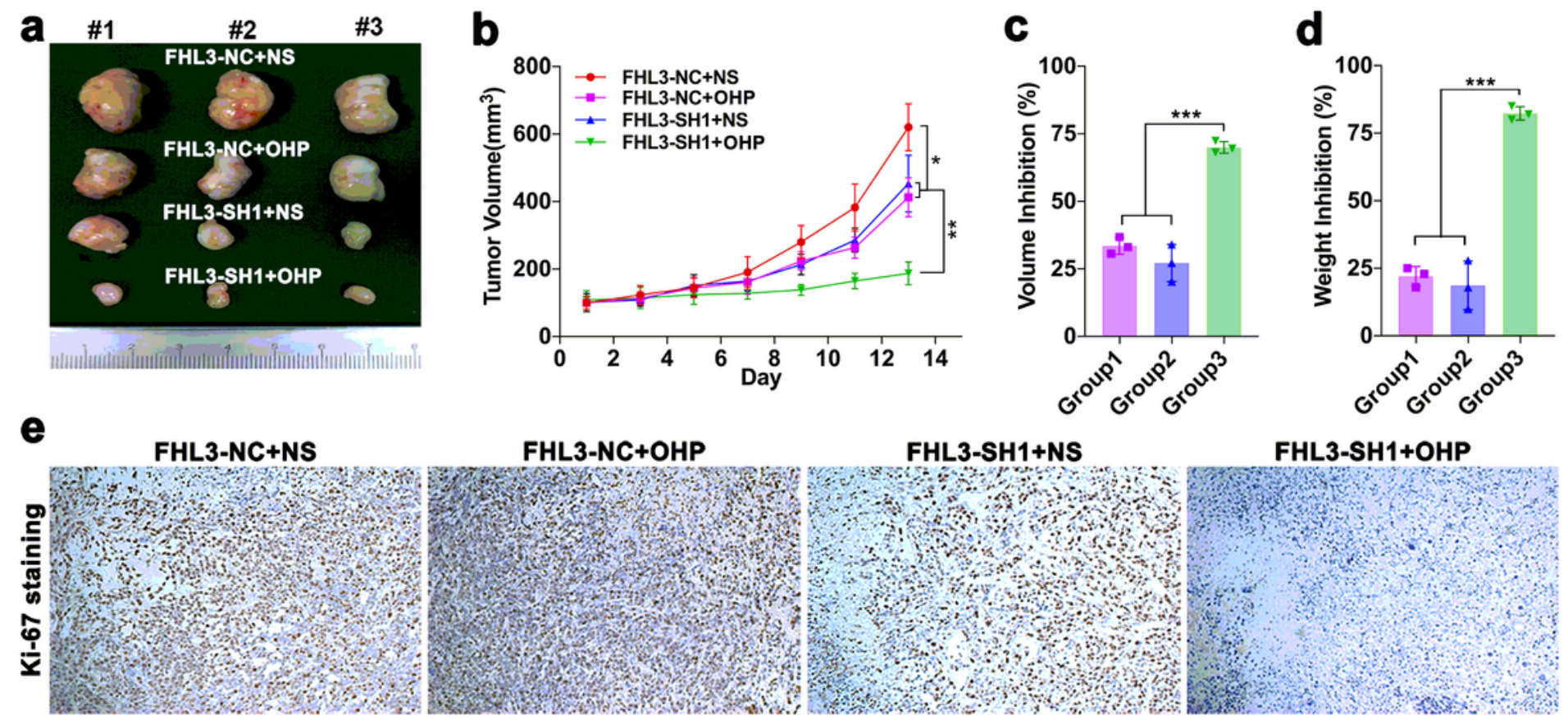

FHL3-NC+OHP

FHL3-SH1+NS

FHL3-SH1+OHP

\section{Figure 5}

The genetic characteristics in FHL3-knockdown-induced epithelial phenotypes. a, b MAPK, PI3K/Akt and TGF $\beta$ pathways is close to FHL3 by KEEG and GO analysis; $1-4$ The activity of MAPK pathway is detected by western blot, and the down-regulation of ERK1/2, Thr202/204-ERK1/2 (p-ERK1/2), pERK1/2/ERK1/2 and Thr180/182-P38 and p-P38/P38 is observed after FHL3 knockdown; d1-4 The activity of PI3K/AKT pathway is detected by western blot, and both of the down-regulation of PI3K-p85, Tyr607-PI3K-p85 (p-PI3K), S473-Akt (p-Akt), p-Akt/Akt, Ser9-GSK3 $\beta$ and Ser9-GSK3ß/GSK3 $\beta$, and upregulation of Akt, Ser217/279-GSK3 $\beta$ and Ser217/279-GSK3 $\beta /$ GSK3 $\beta$ are observed after FHL3 knockdown; e1-4 The activity of TGF $\beta /$ Smad pathway is detected by western blot, and the downregulation of TGF $\beta$, Thr8-Smad2/3 (p-Smad2/3), Ser423/425-Smad2/3 ( $\mathrm{p}-\mathrm{Smad} 2 / 3)$ and $p$ Smad2/3/Smad2/3 is observed after FHL3 knockdown, while the status of Smad4 is not significantly affected by FHL3. 
a

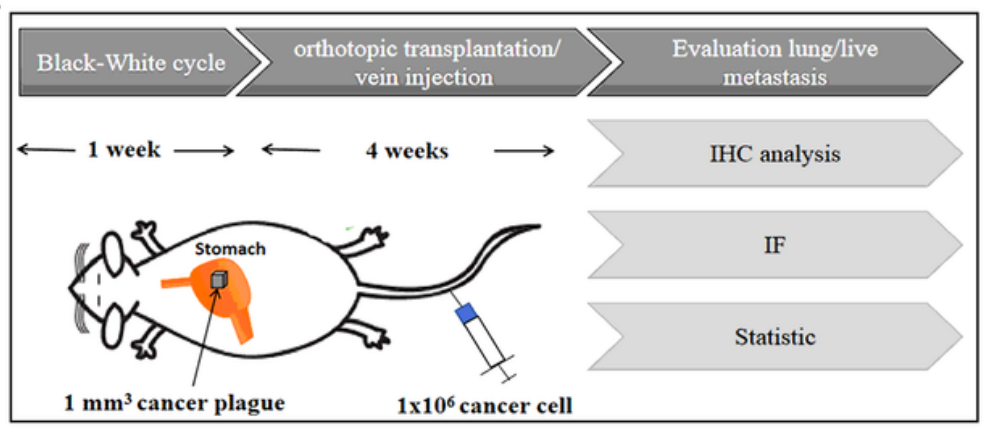

C

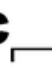

Metastasis in lung

FHL3-NC+OHP

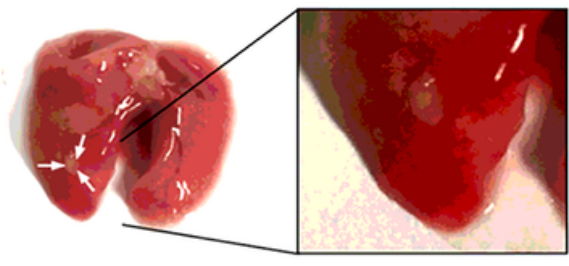

FHL3-SH1+OHP

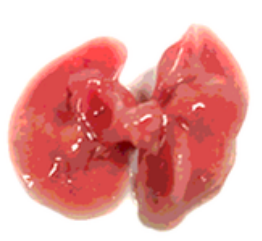

d

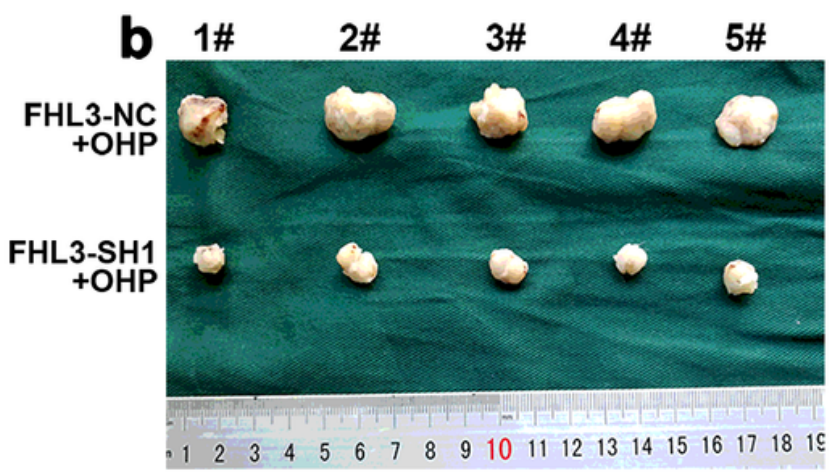

Metastasis in lung

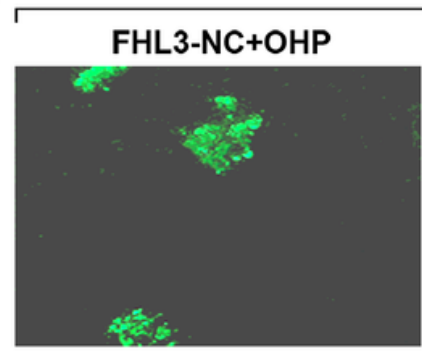

FHL3-SH1+OHP e

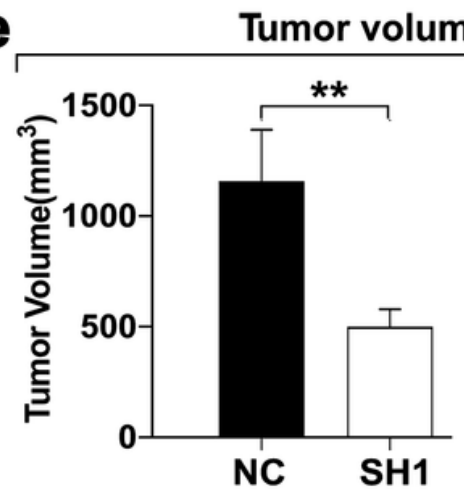

f

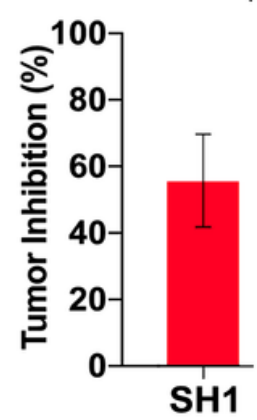

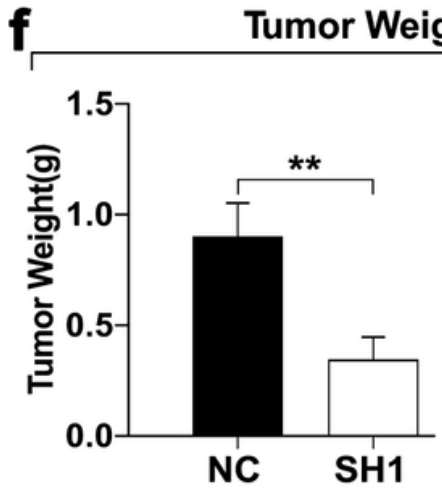

g Metastasis

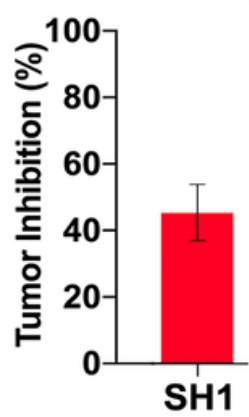

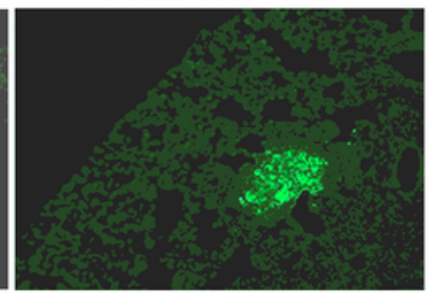

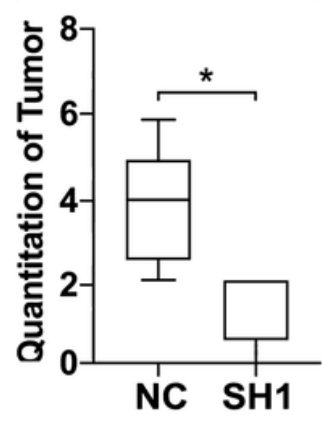

Figure 6

The FHL3-mediated OPH resistance in subcutaneous tumor model. a, b Same volume tumors are transplanted into subcutaneous in 4-weeks-age mouse for 2 weeks, and the tumor volume is detected every one day. c, $\mathrm{d} 2$ weeks later, the mouse is harvested to investigate the tumor volume and weight in vitro; e The ki-67 staining assay is performed. 
a

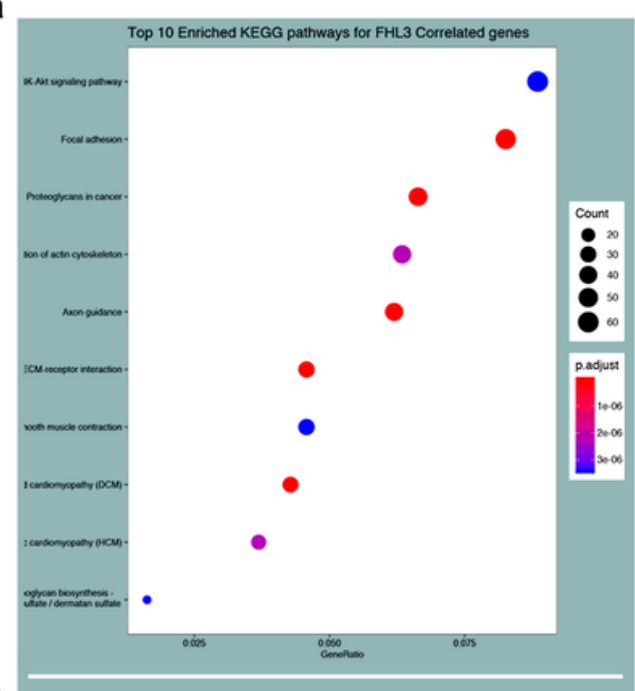

b

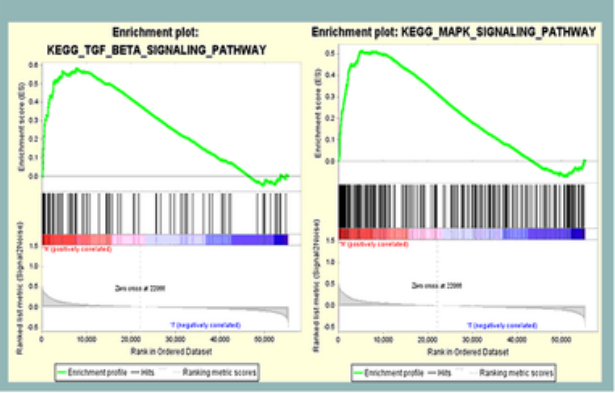

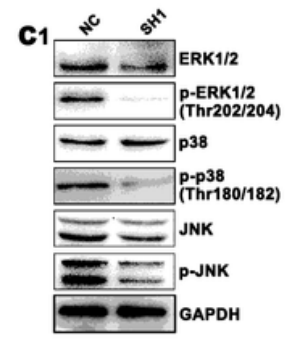
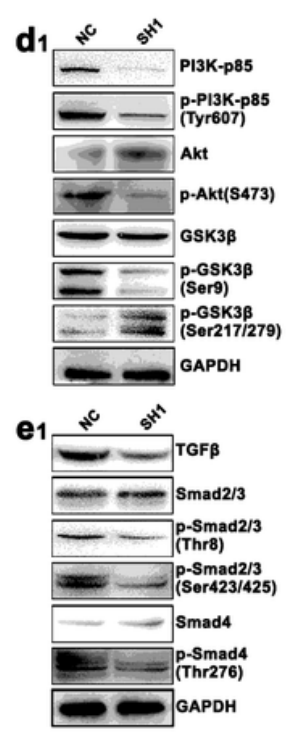

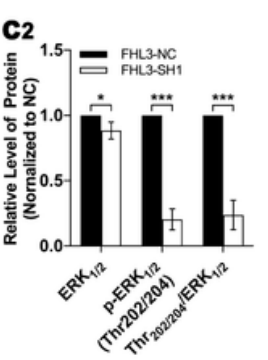

d2
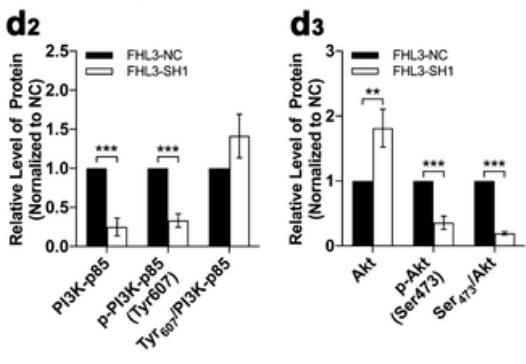

$\mathrm{d}_{4}$

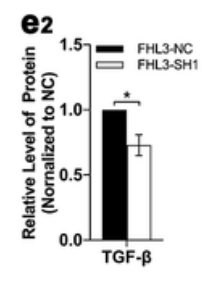

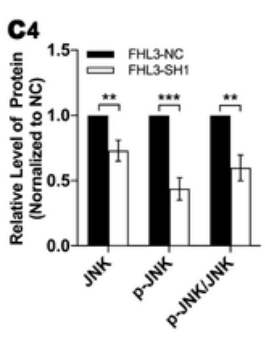

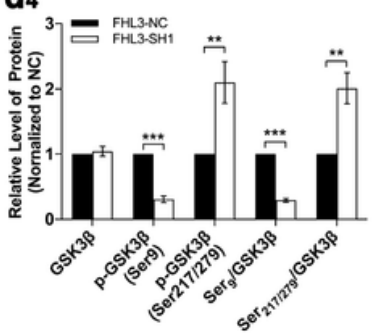

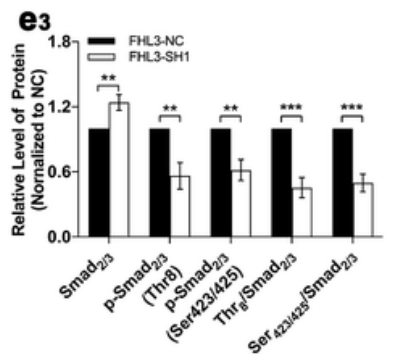

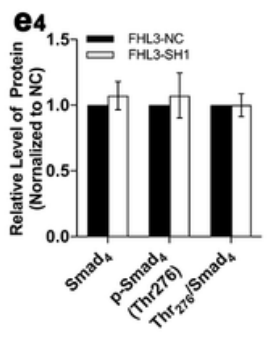

Figure 7

The FHL3-mediated tumor metastasis in orthotopic transplantation model and lung metastasis model. a The schematic diagram of orthotopic transplantation model and lung metastasis model; $b$, e and $f$ Tumor display of orthotopic transplantation model; $\mathrm{d}$, g Metastatic tumors in lungs are displayed in original specimens and frozen slices. 

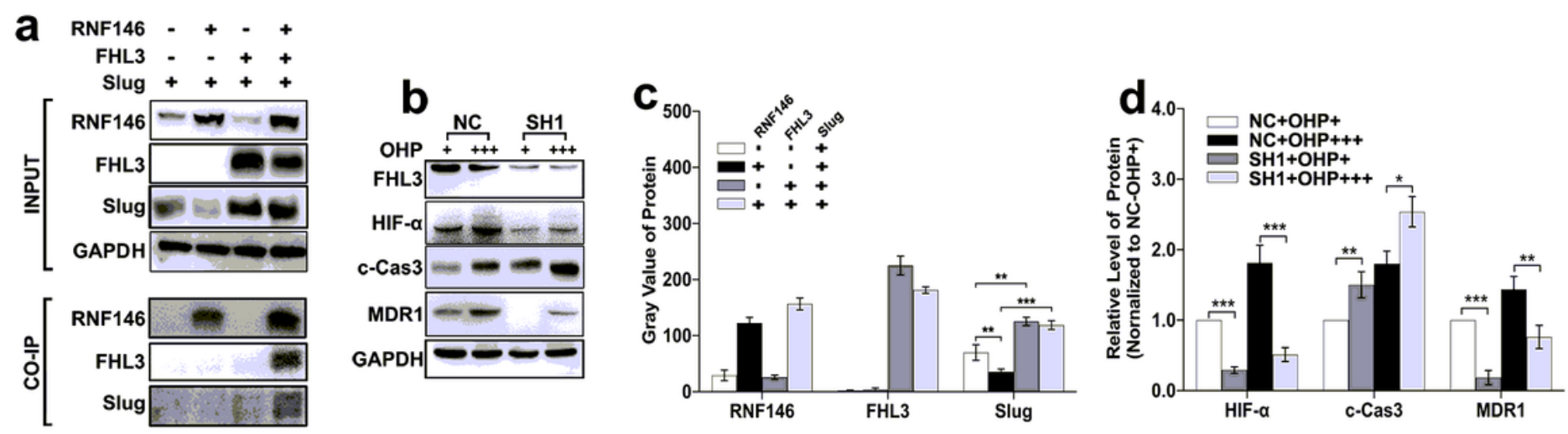

e
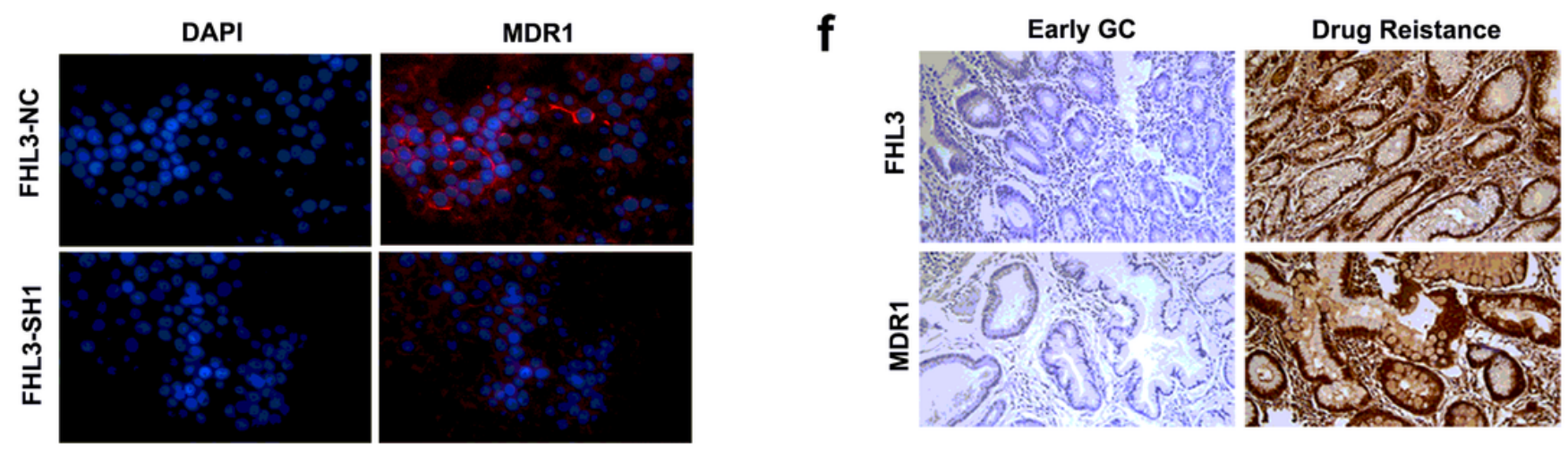

\section{g}
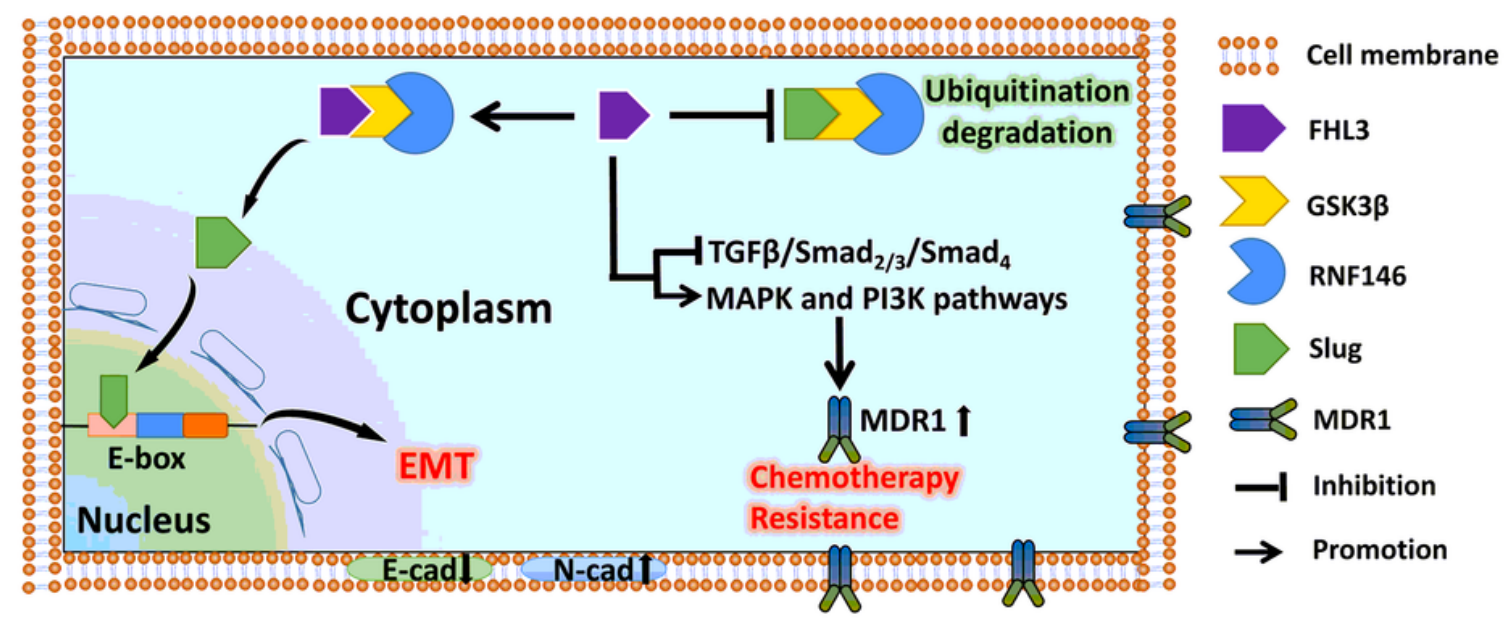

Figure 8

The molecular mechanisms of FHL3-mediated EMT-associated chemotherapy. a, c 293T is transfected with Slug-plasmid and/or RNF146-plasmid and FHL3-plasmid, and the basic up-regulation of those protein is detected by western blot; Using beat-myc detects the interaction between FHL3, Slug and RNF146, and the result shows that RNF146-myc can interact with FHL3-HA and Slug-Flag; $b$, $d$ The levels of HIF-1a, cleaved-caspase-3 and MDR1 are investigated in OPH-resistance FHL3-NC/SH1 groups which are treated with low-dose or high-dose $\mathrm{OPH}$ by western blot. And the results show that high-dose treatment of OPH up-regulates the level of HIF-a, cleaved-caspase-3 and MDR1 in OPH-resistance FHL3NC. HGC. cells when comnare with low-dnse treatment of OPH. In addition, both the down-regulation of Loading [MathJax]/jax/output/CommonHTML/jax.js 
HIF- $\mathrm{a}$ and MDR1, and the up-regulation of cleaved-caspase-3 are observed in OPH-resistance FHL3-SH1 HGC cells when compare with the OPH-resistance FHL3-NC HGC cells; e, $f$ The relationship of FHL3 and MDR1 are investigated in GC cell line and sample slices. IHC shows that advanced GC with chemotherapy resistance have higher level of FHL3 and MDR1. g Mechanism scheme of FHL3 induce EMT and chemotherapy resistance. 